\title{
European Option Pricing Formula in Risk-Aversive Markets
}

\author{
Shujin Wu $(D)$ and Shiyu Wang \\ Key Laboratory of Advanced Theory and Application in Statistics and Data Science, MOE School of Statistics, \\ East China Normal University, Shanghai 200062, China \\ Correspondence should be addressed to Shujin Wu; sjwu@stat.ecnu.edu.cn
}

Received 18 May 2021; Accepted 20 July 2021; Published 31 July 2021

Academic Editor: Bosheng Song

Copyright ( $\odot 2021$ Shujin Wu and Shiyu Wang. This is an open access article distributed under the Creative Commons Attribution License, which permits unrestricted use, distribution, and reproduction in any medium, provided the original work is properly cited.

\begin{abstract}
In this study, using the method of discounting the terminal expectation value into its initial value, the pricing formulas for European options are obtained under the assumptions that the financial market is risk-aversive, the risk measure is standard deviation, and the price process of underlying asset follows a geometric Brownian motion. In particular, assuming the option writer does not need the risk compensation in a risk-neutral market, then the obtained results are degenerated into the famous Black-Scholes model (1973); furthermore, the obtained results need much weaker conditions than those of the Black-Scholes model. As a by-product, the obtained results show that the value of European option depends on the drift coefficient $\mu$ of its underlying asset, which does not display in the Black-Scholes model only because $\mu=r$ in a risk-neutral market according to the no-arbitrage opportunity principle. At last, empirical analyses on Shanghai 50 ETF options and S\&P 500 options show that the fitting effect of obtained pricing formulas is superior to that of the Black-Scholes model.
\end{abstract}

\section{Introduction}

The option pricing theory began in 1900 when the French mathematician Louis Bachelier deduced an option pricing formula under the assumption that underlying asset prices follow a Brownian motion with zero drift. Since then, lots of researchers have contributed to the theory. Black and Scholes [1] present the very famous option pricing formula (i.e., Black-Scholes model) in a risk-neutral market and according to the no-arbitrage opportunity principle. Merton [2] shows the Black-Scholes-type model can be derived from weaker assumptions than in their original formulation and present some pricing methods for non-European options. Bakshi et al. [3] first derive an option pricing model that allows volatility, interest rates, and jumps to be stochastic. Gârleanu et al. [4] model demand-pressure effects on option prices. The model shows that demand pressure in one option contract increases its price by an amount proportional to the variance of the unhedgeable part of the option. Cai and Kou [5] propose a jump diffusion model for asset prices whose jump sizes have a mixed-exponential distribution, which is a weighted average of exponential distributions but with possibly negative weights, and then they extend the analytical tractability of the Black-Scholes model to alternative models. Bernarda and Czadob [6] investigate the pricing of basket options and more generally of complex exotic contracts depending on multiple indices. Their approach assumes that the underlying assets evolve as dependent $\operatorname{GARCH}(1,1)$ processes. The dependence among the assets is modeled using a copula based on pair copula constructions. Bandi and Bertsimas [7] combine robust optimization and the idea of $\varepsilon$-arbitrage to propose a tractable approach to price a wide variety of options. Bao et al. [8] present a method that there is a possibility to get statistical arbitrage from Black-Scholes's option price.

In the last five years, there are still many researchers contributing to the theory of option pricing. Moretto et al. [9] study option pricing under deformed Gaussian distributions. Leippold and Scharer [10] develop a stochastic liquidity model, and they investigate discrete-time option pricing with stochastic liquidity. Hoka and Chanb [11] develop an option pricing method based on Legendre series expansion of the density function, and approximation formulas for pricing European type options are derived. 
Davison and Mamba [12] obtain a solution of the Black-Scholes equation with a nonsmooth boundary condition using symmetry methods. Willems [13] derives a series expansion for the price of a continuously sampled arithmetic Asian option in the Black-Scholes setting. The expansion is based on polynomials that are orthogonal with respect to the log-normal distribution. More literature studies can refer to Liu et al. [14], Friz et al. [15], Dubinsky et al. [16], Huh [17], Liu et al. [18], Siddiqi [19] and their studies.

Although there are a huge number of literature studies on option pricing, they almost assume the financial markets are risk-neutral and complete, especially since Black and Scholes [1]. However, according to the theory and empirical analysis of risk, real financial markets are risk-aversive and incomplete. That is, investors need risk compensation for risky assets, and many risky assets cannot be duplicated by any portfolio constructed in real financial markets.

In this study, using the method of discounting the terminal expectation value into its initial value, we obtain European option pricing formula under the assumptions that the financial market is risk-aversive, the risk measure is standard deviation, and the price process of underlying asset follows a geometric Brownian motion. In particular, if the option writer does not need the risk compensation in a riskneutral market, then our obtained results are degenerated into the Black-Scholes model [1]; furthermore, our obtained results need much weaker conditions than those in the Black-Scholes model. At last, we take the Shanghai 50 ETF options, the first floor option in the Chinese financial market, and S\&P 500 options as samples to compare the fitting effect. The empirical analyses show that the fitting effect of our pricing formulas is superior to that of the Black-Scholes model.

\section{The Black-Scholes Formula}

In this study, we will investigate European option pricing and compare our results with those of Black and Scholes [1]. Thus, we first retell the main results of Black \& Scholes [1].

Black and Scholes [1] present nine assumptions in the market for the security and for the option and then obtain their famous option pricing formula.

Assumption 1. Security price satisfies a geometric Brownian motion (GBM) model, where its drift coefficient and diffusion coefficient are constant through time. That is, the security price satisfies stochastic differential equation:

$$
\frac{\mathrm{d} S_{t}}{S_{t}}=\mu \mathrm{d} t+\sigma \mathrm{d} B_{t}
$$

where $\mu$ and $\sigma$ are constant and $\sigma>0$.

Assumption 2. The short-term interest rate $r$ is known and is constant through time. That is, the risk-free bond price satisfies ordinary differential equation:

$$
\frac{\mathrm{d} P_{t}}{P_{t}}=r \mathrm{~d} t
$$

where $r$ is constant.
Assumption 3. The security pays no dividends or other distributions.

Assumption 4. There are no transaction costs in buying or selling the security or the option.

Assumption 5. The security can be continuously transacted.

Assumption 6. The amount of security can be arbitrarily divided.

Assumption 7. It is possible to borrow any fraction of the price of a security to buy it or to hold it, at the short-term interest rate.

Assumption 8. There are no penalties to short selling. A seller who does not own a security will simply accept the price of the security from a buyer and will agree to settle with the buyer on some future date by paying him an amount equal to the price of the security on that date.

Assumption 9. There is no-arbitrage opportunity.

When the above assumptions all hold, Black and Scholes [1] derived the pricing formula for European options, which is the Black-Scholes model.

Theorem 1 (see [1]). If Assumptions 1 to 9 hold, then the values of European call option and European put option follow as

$$
\begin{aligned}
& C\left(S_{0}, K, r, \sigma, \tau\right)=S_{0} \Phi\left(d_{2}\right)-K e^{-r \tau} \Phi\left(d_{1}\right), \\
& P\left(S_{0}, K, r, \sigma, \tau\right)=K e^{-r \tau} \Phi\left(-d_{1}\right)-S_{0} \Phi\left(-d_{2}\right),
\end{aligned}
$$

where $S_{0}$ is the initial price of underlying asset, $K$ is the strike price of option, $r$ is the short-term interest rate, $\sigma$ is the diffusion coefficient of underlying asset, $\tau$ is the left expiration time of option, $\Phi(\cdot)$ is the cumulative density function of standard normal distribution, and

$$
\begin{aligned}
& d_{1}=\frac{1}{\sigma \sqrt{\tau}}\left(\ln \frac{S_{0}}{K}+\left(r-\frac{1}{2} \sigma^{2}\right) \tau\right), \\
& d_{2}=d_{1}+\sigma \sqrt{\tau} .
\end{aligned}
$$

\section{European Option Pricing in Risk- Aversive Markets}

Black-Scholes model and its modified versions have some defects. In fact, because real financial markets are incomplete, an option may not be duplicated constantly, so its value deduced by the asset duplication method and noarbitrage principle may lose the deductive basis. On the other hand, real financial markets are risk-aversive. Option seller undertakes the total risk and option buyer has no any risk, so option seller needs a reasonable risk compensation according to the theory of risk. In this section, we will deduce the option pricing formula in risk-aversive markets only under three assumptions, i.e., Assumptions 1, 2, and 4 in 
Section 2, which is reasonably far more than the Black-Scholes model and its modified versions.

In risk-aversive markets, assume the price process of some risky asset $X$ by $\left\{X_{t}, t \geq 0\right\}$. Then the value of European call option at expiration time $T$ with underlying asset $X$ and strike price $K$ follows as

$$
\left(X_{T}-K\right)^{+}
$$

here and in the sequel the operator $(\cdot)^{+}=\max \{0, \cdot\}$. The value of European put option at expiration time $T$ with underlying asset $X$ and strike price $K$ follows as

$$
\left(K-X_{T}\right)^{+} \text {. }
$$

Note that no matter call option or put option, it is always its seller undertakes the total risk and its buyer has no any risk. According to risk theory, the seller reasonably requires some risk compensation $\lambda \rho(X)$, where $\lambda \geq 0$ is the riskcompensation coefficient, $\rho(\cdot)$ is the risk measure, and $X$ is the risk size. After the seller has received the reasonable risk compensation, the seller takes risky asset as equivalent riskfree bond, so it follows from (6) that the value at time $t$ of European call option with underlying asset $X$, strike price $K$, and expiration time $T$ follows as

$$
\begin{aligned}
C_{t}(X, K, T, r, \lambda)= & e^{-r(T-t)}\left\{E\left[\left(X_{T}-K\right)^{+}\right]\right. \\
& \left.+\lambda \rho\left(\left(X_{T}-K\right)^{+}\right)\right\}, \quad \forall t \leq T,
\end{aligned}
$$

where $r \geq 0$ is the risk-free rate during $[t, T]$. Analogically, it follows from (7) that the value of European put option with underlying asset $X$, strike price $K$, and expiration time $T$ follows as

$$
\begin{aligned}
P_{t}(X, K, T, r, \lambda)= & e^{-r(T-t)}\left\{E\left[\left(K-X_{T}\right)^{+}\right]\right. \\
& \left.+\lambda \rho\left(\left(K-X_{T}\right)^{+}\right)\right\}, \quad \forall t \leq T .
\end{aligned}
$$

In conclusion, we obtain the following proposition from (8) and (9).

Proposition 1. In risk-aversive market, assume that the risk measure is $\rho(\cdot)$ and the risk-compensation coefficient is $\lambda \geq 0$, and assuming a European option with underlying asset $X$, strike price $K$, and expiration time $T$, then its call-option value at time $t$ follows as

$C_{t}(X, K, T, r, \lambda)=e^{-r(T-t)}\left\{E\left[\left(X_{T}-K\right)^{+}\right]+\lambda \rho\left(\left(X_{T}-K\right)^{+}\right)\right\}$,

and its put-option value at time $t$ follows as

$$
P_{t}(X, K, T, r, \lambda)=e^{-r(T-t)}\left\{E\left[\left(K-X_{T}\right)^{+}\right]+\lambda \rho\left(\left(K-X_{T}\right)^{+}\right)\right\},
$$

where $r \geq 0$ is the risk-free rate during $[t, T]$ and $t \leq T$.

In order to obtain a closed-form solution to Proposition 1 , in the following, we always assume the price process of underlying asset follows some geometric Brownian motion model (1), and the risk measure is the standard deviation, i.e., $\rho(Z)=\operatorname{std}(Z)$ for any risk variable $Z$.

Using Proposition 1, we can deduce the value of European call option in risk-aversive markets and under Assumptions 1,2 , and 4 .

Theorem 2. In a risk-aversive market, assuming that underlying asset follows a geometric Brownian motion with drift coefficient $\mu$ and diffusion coefficient $\sigma>0$, the current price of underlying asset is $S_{0}$, risk-free interest rate is $r$ through the time, the risk-compensation factor is $\lambda \geq 0$, and the risk measure is standard deviation, then the value of European call option with strike price $K$ and left expiration time $\tau$ follows as

$$
\begin{aligned}
C\left(S_{0}, K, r, \sigma, \tau, \lambda\right)= & S_{0} e^{(\mu-r) \tau} \Phi\left(d_{2}\right)-K e^{-r \tau} \Phi\left(d_{1}\right)+\lambda e^{-r \tau} \cdot \operatorname{sqrt}\left\{S_{0}^{2} e^{2 \mu \tau}\left(e^{\sigma^{2} \tau} \Phi\left(d_{3}\right)-\Phi^{2}\left(d_{2}\right)\right)-K S_{0} e^{\mu \tau} \Phi\left(d_{2}\right) \Phi\left(-d_{1}\right)\right. \\
& \left.+K^{2} \Phi\left(d_{1}\right) \Phi\left(-d_{1}\right)\right\}
\end{aligned}
$$

where

$$
\begin{aligned}
& d_{1}=\frac{1}{\sigma \sqrt{\tau}}\left(\ln \frac{S_{0}}{K}+\left(\mu-\frac{1}{2} \sigma^{2}\right) \tau\right), \\
& d_{m}=d_{1}+(m-1) \sigma \sqrt{\tau}, \quad m=2,3 .
\end{aligned}
$$

The Proof of Theorem 2 refers to Appendix A.

If a financial market is risk-neutral, then investors treat expected return and deterministic return equally, so expectation yield $\mu$ equals risk-free yield $r$, that is, $\mu=r$. Otherwise, if $\mu \neq r$, there will exist arbitrage opportunity. Furthermore, in a risk-neutral financial market, the risk-

compensation factor equals zero, that is, $\lambda=0$. Thus, it yields from Theorem 1 that we have the following corollary.

Corollary 1. In a risk-neutral market, assuming that underlying asset follows a geometric Brownian motion with drift coefficient $\mu$ and diffusion coefficient $\sigma>0$, the current price of underlying asset is $S_{0}$, and risk-free yield is $r$ through the time, then the value of European call option with strike price $K$ and left expiration time $\tau$ follows as

$$
C\left(S_{0}, K, r, \sigma, \tau\right)=S_{0} \Phi\left(d_{2}\right)-K e^{-r \tau} \Phi\left(d_{1}\right)
$$

where 


$$
\begin{aligned}
& d_{1}=\frac{1}{\sigma \sqrt{\tau}}\left(\ln \frac{S_{0}}{K}+\left(r-\frac{1}{2} \sigma^{2}\right) \tau\right), \\
& d_{2}=d_{1}+\sigma \sqrt{\tau}
\end{aligned}
$$

In the following, we will deduce the pricing formula of European put option. Using Proposition 1, we will construct the pricing formula of European put option in risk-aversive markets and under Assumptions 1, 2, and 4.
Theorem 3. In a risk-aversive market, assuming that underlying asset follows a geometric Brownian motion with drift coefficient $\mu$ and diffusion coefficient $\sigma>0$, the current price of underlying asset is $S_{0}$, risk-free yield is $r$ through the time, the risk-compensation factor is $\lambda \geq 0$, and the risk measure is standard deviation, then the value of European put option with strike price $K$ and left expiration time $\tau$ follows as

$$
\begin{aligned}
P\left(S_{0}, K, r, \sigma, \tau, \lambda\right)= & K e^{-r \tau} \Phi\left(-d_{1}\right)-S_{0} e^{(\mu-r) \tau} \Phi\left(-d_{2}\right)+\lambda e^{-r \tau} \cdot \operatorname{sqrt}\left\{S_{0}^{2} e^{2 \mu \tau}\left(e^{\sigma^{2} \tau} \Phi\left(-d_{3}\right)-\Phi^{2}\left(-d_{2}\right)\right)-K S_{0} e^{\mu \tau} \Phi\left(-d_{2}\right) \Phi\left(d_{1}\right)\right. \\
& \left.+K^{2} \Phi\left(-d_{1}\right) \Phi\left(d_{1}\right)\right\}
\end{aligned}
$$

where

$$
\begin{aligned}
& d_{1}=\frac{1}{\sigma \sqrt{\tau}}\left(\ln \frac{S_{0}}{K}+\left(\mu-\frac{1}{2} \sigma^{2}\right) \tau\right) \\
& d_{m}=d_{1}+(m-1) \sigma \sqrt{\tau}, \quad m=2,3 .
\end{aligned}
$$

The Proof of Theorem 3 refers to Appendix B.

According to the analysis before Corollary 1, if a financial market is risk-neutral, then $\mu=r$ and $\lambda=0$. Furthermore, it yields from Theorem 3 that we have the following corollary.

Corollary 2. In a risk-neutral market, assuming that underlying asset follows a geometric Brownian motion with drift coefficient $\mu$ and diffusion coefficient $\sigma>0$, the current price of underlying asset is $S_{0}$, and risk-free yield is $r$ through the time, then the value of European put option with strike price $K$ and left expiration time $\tau$ follows as

$$
P\left(S_{0}, K, r, \sigma, \tau\right)=K e^{-r \tau} \Phi\left(-d_{1}\right)-S_{0} \Phi\left(-d_{2}\right),
$$

where

$$
\begin{aligned}
& d_{1}=\frac{1}{\sigma \sqrt{\tau}}\left(\ln \frac{S_{0}}{K}+\left(r-\frac{1}{2} \sigma^{2}\right) \tau\right), \\
& d_{2}=d_{1}+\sigma \sqrt{\tau} .
\end{aligned}
$$

Remark 1. Although Corollaries 1 and 2 obtain the same values as those in Theorem 1 for European call option and European put option, Corollaries 1 and 2 need much weaker conditions than those of Theorem 1. That is, Corollaries 1 and 2 improve Theorem 1.

In fact, if Assumptions 1, 2, and 4 in Section 2 hold, Corollaries 1 and 2 hold. However, the conditions that Theorem 1 (i.e., the Black-Scholes model) holds are Assumptions 1 to 9 in Section 2 .

\section{Empirical Analysis of Shanghai 50 ETF Options}

In the section, we will present empirical analysis on Shanghai 50 ETF options, the first floor option in the Chinese financial market, and use the data of September 3 and 4, 2018, to compare the fitting effect of our pricing model and the Black-Scholes pricing model. All used data come from the CSMAR Database, which includes actual option price $C$ or $P$, trading date $t$, exercise date $T$, strike price $K$, and current price of underlying asset $S_{0}$. In addition, the database also includes the historical volatility of Shanghai 50 ETF $\sigma=$ 0.1994 on September 3, 2018, and $\sigma=0.2005$ on September 4,2018 , and the 1-year deposit benchmark interest rate of Chinese Central Bank $r=1.5 \%$, which is chosen as the reference level of risk-free interest rate in the Chinese financial market. All data analyses in the following are worked out by the software MATLAB R2018b.

4.1. Parameter Estimation. There are 48 call options on September 3, 2018, and the current price of underlying asset $S_{0}=2.512$. We take the annual average return rate of the last month as the drift coefficient of the underlying asset $\mu$. According to simple computation, we obtain $\mu=2.557 \%$ and the left expiration time $\tau=(T-t) / 365$ (years), where $t=2018 / 09 / 03$, and then we work out $d_{1}, d_{2}$, and $d_{3}$ by Theorem 2; see Table 1 for detailed data, where $C$ is the actual closing price of call option, $T$ is the expiration time of call option, $K$ is the strike price of call option, $S_{0}$ is the initial price of underlying asset, $\tau$ is the left expiration time of call option, and $d_{1}, d_{2}$, and $d_{3}$ are the parameters in Theorem 2 . Furthermore, we obtain the estimated value of the riskcompensation factor $\lambda=0.0077963$ by the least square method with $R^{2}=0.7058$

There are 60 put options on September 3, 2018, and the current price of underlying asset $S_{0}=2.512$. Similarly, we obtain $\mu=2.93 \%$ and the left expiration time $\tau=(T-t) / 365$ (years), where $t=2018 / 09 / 03$, and then we work out $d_{1}, d_{2}$, and $d_{3}$ by Theorem 3 ; see Table 2 for detailed data, where $P$ is 
TABLe 1: Parameter estimation of $d_{1}, d_{2}$, and $d_{3}$ for call options.

\begin{tabular}{|c|c|c|c|c|c|c|c|}
\hline$C$ & $T$ & $K$ & $S_{0}$ & $\tau$ & $d_{1}$ & $d_{2}$ & $d_{3}$ \\
\hline 0.0030 & $2018 / 09 / 26$ & 2.75 & 2.512 & 0.0630 & -1.8013 & -1.7512 & -1.7012 \\
\hline 0.0073 & $2018 / 09 / 26$ & 2.70 & 2.512 & 0.0630 & -1.4347 & -1.3847 & -1.3346 \\
\hline 0.0146 & $2018 / 09 / 26$ & 2.65 & 2.512 & 0.0630 & -1.0613 & -1.0112 & -0.9612 \\
\hline 0.0150 & $2018 / 10 / 24$ & 2.75 & 2.512 & 0.1397 & -1.2038 & -1.1293 & -1.0547 \\
\hline 0.0159 & $2018 / 12 / 26$ & 2.95 & 2.512 & 0.3123 & -1.4263 & -1.3149 & -1.2035 \\
\hline 0.0220 & $2018 / 12 / 26$ & 2.90 & 2.512 & 0.3123 & -1.2729 & -1.1615 & -1.0501 \\
\hline 0.0228 & $2018 / 10 / 24$ & 2.70 & 2.512 & 0.1397 & -0.9576 & -0.8831 & -0.8086 \\
\hline 0.0275 & $2018 / 09 / 26$ & 2.60 & 2.512 & 0.0630 & -0.6807 & -0.6307 & -0.5806 \\
\hline 0.0287 & $2018 / 12 / 26$ & 2.85 & 2.512 & 0.3123 & -1.1169 & -1.0054 & -0.8940 \\
\hline 0.0350 & $2018 / 10 / 24$ & 2.65 & 2.512 & 0.1397 & -0.7068 & -0.6323 & -0.5578 \\
\hline 0.0372 & $2018 / 12 / 26$ & 2.80 & 2.512 & 0.3123 & -0.9580 & -0.8466 & -0.7352 \\
\hline 0.0451 & $2018 / 09 / 26$ & 2.55 & 2.512 & 0.0630 & -0.2928 & -0.2427 & -0.1927 \\
\hline 0.0484 & $2018 / 12 / 26$ & 2.75 & 2.512 & 0.3123 & -0.7964 & -0.6849 & -0.5735 \\
\hline 0.0500 & $2018 / 10 / 24$ & 2.60 & 2.512 & 0.1397 & -0.4513 & -0.3767 & -0.3022 \\
\hline 0.0601 & $2018 / 12 / 26$ & 2.70 & 2.512 & 0.3123 & -0.6317 & -0.5203 & -0.4088 \\
\hline 0.0695 & $2018 / 09 / 26$ & 2.50 & 2.512 & 0.0630 & 0.1028 & 0.1529 & 0.2029 \\
\hline 0.0698 & $2018 / 10 / 24$ & 2.55 & 2.512 & 0.1397 & -0.1908 & -0.1162 & -0.0417 \\
\hline 0.0742 & $2019 / 03 / 27$ & 2.80 & 2.512 & 0.5616 & -0.7049 & -0.5555 & -0.4061 \\
\hline 0.0756 & $2018 / 12 / 26$ & 2.65 & 2.512 & 0.3123 & -0.4640 & -0.3525 & -0.2411 \\
\hline 0.0878 & $2019 / 03 / 27$ & 2.75 & 2.512 & 0.5616 & -0.5844 & -0.4349 & -0.2855 \\
\hline 0.0938 & $2018 / 12 / 26$ & 2.60 & 2.512 & 0.3123 & -0.2930 & -0.1816 & -0.0701 \\
\hline 0.0944 & $2018 / 10 / 24$ & 2.50 & 2.512 & 0.1397 & 0.0749 & 0.1495 & 0.2240 \\
\hline 0.0980 & $2018 / 09 / 26$ & 2.45 & 2.512 & 0.0630 & 0.5064 & 0.5565 & 0.6066 \\
\hline 0.1026 & $2019 / 03 / 27$ & 2.70 & 2.512 & 0.5616 & -0.4616 & -0.3121 & -0.1627 \\
\hline 0.1149 & $2018 / 12 / 26$ & 2.55 & 2.512 & 0.3123 & -0.1188 & -0.0073 & 0.1041 \\
\hline 0.1190 & $2019 / 03 / 27$ & 2.65 & 2.512 & 0.5616 & -0.3365 & -0.1870 & -0.0376 \\
\hline 0.1247 & $2018 / 10 / 24$ & 2.45 & 2.512 & 0.1397 & 0.3460 & 0.4205 & 0.4950 \\
\hline 0.1361 & $2018 / 09 / 26$ & 2.40 & 2.512 & 0.0630 & 0.9184 & 0.9684 & 1.0185 \\
\hline 0.1380 & $2019 / 03 / 27$ & 2.60 & 2.512 & 0.5616 & -0.2090 & -0.0596 & 0.0899 \\
\hline 0.1390 & $2018 / 12 / 26$ & 2.50 & 2.512 & 0.3123 & 0.0589 & 0.1704 & 0.2818 \\
\hline 0.1592 & $2018 / 10 / 24$ & 2.40 & 2.512 & 0.1397 & 0.6226 & 0.6971 & 0.7717 \\
\hline 0.1623 & $2019 / 03 / 27$ & 2.55 & 2.512 & 0.5616 & -0.0791 & 0.0704 & 0.2198 \\
\hline 0.1671 & $2018 / 12 / 26$ & 2.45 & 2.512 & 0.3123 & 0.2402 & 0.3517 & 0.4631 \\
\hline 0.1770 & $2018 / 09 / 26$ & 2.35 & 2.512 & 0.0630 & 1.3390 & 1.3891 & 1.4391 \\
\hline 0.1861 & $2019 / 03 / 27$ & 2.50 & 2.512 & 0.5616 & 0.0534 & 0.2029 & 0.3523 \\
\hline 0.1968 & $2018 / 10 / 24$ & 2.35 & 2.512 & 0.1397 & 0.9051 & 0.9796 & 1.0541 \\
\hline 0.1981 & $2018 / 12 / 26$ & 2.40 & 2.512 & 0.3123 & 0.4253 & 0.5367 & 0.6481 \\
\hline 0.2110 & $2019 / 03 / 27$ & 2.45 & 2.512 & 0.5616 & 0.1886 & 0.3381 & 0.4875 \\
\hline 0.2209 & $2018 / 09 / 26$ & 2.30 & 2.512 & 0.0630 & 1.7687 & 1.8187 & 1.8688 \\
\hline 0.2297 & $2018 / 12 / 26$ & 2.35 & 2.512 & 0.3123 & 0.6142 & 0.7256 & 0.8371 \\
\hline 0.2360 & $2018 / 10 / 24$ & 2.30 & 2.512 & 0.1397 & 1.1936 & 1.2681 & 1.3427 \\
\hline 0.2421 & $2019 / 03 / 27$ & 2.40 & 2.512 & 0.5616 & 0.3266 & 0.4761 & 0.6255 \\
\hline 0.2660 & $2018 / 12 / 26$ & 2.30 & 2.512 & 0.3123 & 0.8072 & 0.9186 & 1.0300 \\
\hline 0.2704 & $2018 / 09 / 26$ & 2.25 & 2.512 & 0.0630 & 2.2078 & 2.2578 & 2.3079 \\
\hline 0.2767 & $2019 / 03 / 27$ & 2.35 & 2.512 & 0.5616 & 0.4675 & 0.6169 & 0.7664 \\
\hline 0.3074 & $2018 / 12 / 26$ & 2.25 & 2.512 & 0.3123 & 1.0044 & 1.1158 & 1.2273 \\
\hline 0.3163 & $2018 / 09 / 26$ & 2.20 & 2.512 & 0.0630 & 2.6567 & 2.7068 & 2.7568 \\
\hline 0.3450 & $2019 / 03 / 27$ & 2.25 & 2.512 & 0.5616 & 0.7585 & 0.9079 & 1.0574 \\
\hline
\end{tabular}

the actual closing price of put option, $T$ is the expiration time of put option, $K$ is the strike price of put option, $S_{0}$ is the initial price of underlying asset, $\tau$ is the left expiration time of put option, and $d_{1}, d_{2}$, and $d_{3}$ are the parameters in Theorem 3. Furthermore, we obtain the estimated value of the risk-compensation factor $\lambda=0.013306$ by the least square method with $R^{2}=0.6218$.

In addition, we obtain the estimation of risk-free rate $r=1.768 \%$ by minimizing the mean square error of the Black-Scholes model on September 3, 2018. Note that $r=$
$1.768 \%$ is a little higher than the 1 -year deposit benchmark interest rate of Chinese Central Bank $1.5 \%$, so it is very reasonable that we take $r=1.768 \%$ as the risk-free rate. Furthermore, it is far advantageous for the Black-Scholes model to improve its fitting effect.

4.2. Comparison of Pricing Effect. In the section, we will compare the fitting effect of our pricing formulas with the Black-Scholes model. 
TABLe 2: Parameter estimation of $d_{1}, d_{2}$, and $d_{3}$ for put options.

\begin{tabular}{|c|c|c|c|c|c|c|c|}
\hline$P$ & $T$ & $K$ & $S_{0}$ & $\tau$ & $d_{1}$ & $d_{2}$ & $d_{3}$ \\
\hline 0.0015 & $2018 / 09 / 26$ & 2.20 & 2.512 & 0.0630 & 2.6567 & 2.7068 & 2.7568 \\
\hline 0.0027 & $2018 / 09 / 26$ & 2.25 & 2.512 & 0.0630 & 2.2078 & 2.2578 & 2.3079 \\
\hline 0.0055 & $2018 / 09 / 26$ & 2.30 & 2.512 & 0.0630 & 1.7687 & 1.8187 & 1.8688 \\
\hline 0.0115 & $2018 / 09 / 26$ & 2.35 & 2.512 & 0.0630 & 1.3390 & 1.3891 & 1.4391 \\
\hline 0.0165 & $2018 / 10 / 24$ & 2.30 & 2.512 & 0.1397 & 1.1936 & 1.2681 & 1.3427 \\
\hline 0.0205 & $2018 / 09 / 26$ & 2.40 & 2.512 & 0.0630 & 0.9184 & 0.9684 & 1.0185 \\
\hline 0.0247 & $2018 / 12 / 26$ & 2.20 & 2.512 & 0.3123 & 1.2061 & 1.3175 & 1.4289 \\
\hline 0.0255 & $2018 / 10 / 24$ & 2.35 & 2.512 & 0.1397 & 0.9051 & 0.9796 & 1.0541 \\
\hline 0.0324 & $2018 / 12 / 26$ & 2.25 & 2.512 & 0.3123 & 1.0044 & 1.1158 & 1.2273 \\
\hline 0.0343 & $2018 / 09 / 26$ & 2.45 & 2.512 & 0.0630 & 0.5064 & 0.5565 & 0.6066 \\
\hline 0.0371 & $2018 / 10 / 24$ & 2.40 & 2.512 & 0.1397 & 0.6226 & 0.6971 & 0.7717 \\
\hline 0.0432 & $2018 / 12 / 26$ & 2.30 & 2.512 & 0.3123 & 0.8072 & 0.9186 & 1.0300 \\
\hline 0.0528 & $2018 / 10 / 24$ & 2.45 & 2.512 & 0.1397 & 0.3460 & 0.4205 & 0.4950 \\
\hline 0.0531 & $2018 / 09 / 26$ & 2.50 & 2.512 & 0.0630 & 0.1028 & 0.1529 & 0.2029 \\
\hline 0.0565 & $2018 / 12 / 26$ & 2.35 & 2.512 & 0.3123 & 0.6142 & 0.7256 & 0.8371 \\
\hline 0.0608 & $2019 / 03 / 27$ & 2.25 & 2.512 & 0.5616 & 0.7585 & 0.9079 & 1.0574 \\
\hline 0.0730 & $2019 / 03 / 27$ & 2.30 & 2.512 & 0.5616 & 0.6114 & 0.7609 & 0.9103 \\
\hline 0.0733 & $2018 / 10 / 24$ & 2.50 & 2.512 & 0.1397 & 0.0749 & 0.1495 & 0.2240 \\
\hline 0.0734 & $2018 / 12 / 26$ & 2.40 & 2.512 & 0.3123 & 0.4253 & 0.5367 & 0.6481 \\
\hline 0.0791 & $2018 / 09 / 26$ & 2.55 & 2.512 & 0.0630 & -0.2928 & -0.2427 & -0.1927 \\
\hline 0.0902 & $2019 / 03 / 27$ & 2.35 & 2.512 & 0.5616 & 0.4675 & 0.6169 & 0.7664 \\
\hline 0.0920 & $2018 / 12 / 26$ & 2.45 & 2.512 & 0.3123 & 0.2402 & 0.3517 & 0.4631 \\
\hline 0.0989 & $2018 / 10 / 24$ & 2.55 & 2.512 & 0.1397 & -0.1908 & -0.1162 & -0.0417 \\
\hline 0.1074 & $2019 / 03 / 27$ & 2.40 & 2.512 & 0.5616 & 0.3266 & 0.4761 & 0.6255 \\
\hline 0.1117 & $2018 / 09 / 26$ & 2.60 & 2.512 & 0.0630 & -0.6807 & -0.6307 & -0.5806 \\
\hline 0.1140 & $2018 / 12 / 26$ & 2.50 & 2.512 & 0.3123 & 0.0589 & 0.1704 & 0.2818 \\
\hline 0.1244 & $2019 / 03 / 27$ & 2.45 & 2.512 & 0.5616 & 0.1886 & 0.3381 & 0.4875 \\
\hline 0.1284 & $2018 / 10 / 24$ & 2.60 & 2.512 & 0.1397 & -0.4513 & -0.3767 & -0.3022 \\
\hline 0.1401 & $2018 / 12 / 26$ & 2.55 & 2.512 & 0.3123 & -0.1188 & -0.0073 & 0.1041 \\
\hline 0.1483 & $2019 / 03 / 27$ & 2.50 & 2.512 & 0.5616 & 0.0534 & 0.2029 & 0.3523 \\
\hline 0.1498 & $2018 / 09 / 26$ & 2.65 & 2.512 & 0.0630 & -1.0613 & -1.0112 & -0.9612 \\
\hline 0.1686 & $2018 / 12 / 26$ & 2.60 & 2.512 & 0.3123 & -0.2930 & -0.1816 & -0.0701 \\
\hline 0.1750 & $2019 / 03 / 27$ & 2.55 & 2.512 & 0.5616 & -0.0791 & 0.0704 & 0.2198 \\
\hline 0.1908 & $2018 / 09 / 26$ & 2.70 & 2.512 & 0.0630 & -1.4347 & -1.3847 & -1.3346 \\
\hline 0.2003 & $2018 / 12 / 26$ & 2.65 & 2.512 & 0.3123 & -0.4640 & -0.3525 & -0.2411 \\
\hline 0.2007 & $2018 / 10 / 24$ & 2.70 & 2.512 & 0.1397 & -0.9576 & -0.8831 & -0.8086 \\
\hline 0.2009 & $2019 / 03 / 27$ & 2.60 & 2.512 & 0.5616 & -0.2090 & -0.0596 & 0.0899 \\
\hline 0.2278 & $2018 / 12 / 26$ & 2.70 & 2.512 & 0.3123 & -0.6317 & -0.5203 & -0.4088 \\
\hline 0.2312 & $2019 / 03 / 27$ & 2.65 & 2.512 & 0.5616 & -0.3365 & -0.1870 & -0.0376 \\
\hline 0.2370 & $2018 / 09 / 26$ & 2.75 & 2.512 & 0.0630 & -1.8013 & -1.7512 & -1.7012 \\
\hline 0.2440 & $2018 / 10 / 24$ & 2.75 & 2.512 & 0.1397 & -1.2038 & -1.1293 & -1.0547 \\
\hline 0.2635 & $2019 / 03 / 27$ & 2.70 & 2.512 & 0.5616 & -0.4616 & -0.3121 & -0.1627 \\
\hline 0.2657 & $2018 / 12 / 26$ & 2.75 & 2.512 & 0.3123 & -0.7964 & -0.6849 & -0.5735 \\
\hline 0.2828 & $2018 / 09 / 26$ & 2.80 & 2.512 & 0.0630 & -2.1613 & -2.1112 & -2.0612 \\
\hline 0.2976 & $2019 / 03 / 27$ & 2.75 & 2.512 & 0.5616 & -0.5844 & -0.4349 & -0.2855 \\
\hline 0.3089 & $2018 / 12 / 26$ & 2.80 & 2.512 & 0.3123 & -0.9580 & -0.8466 & -0.7352 \\
\hline 0.3323 & $2018 / 09 / 26$ & 2.85 & 2.512 & 0.0630 & -2.5149 & -2.4648 & -2.4148 \\
\hline 0.3330 & $2019 / 03 / 27$ & 2.80 & 2.512 & 0.5616 & -0.7049 & -0.5555 & -0.4061 \\
\hline 0.3477 & $2018 / 12 / 26$ & 2.85 & 2.512 & 0.3123 & -1.1169 & -1.0054 & -0.8940 \\
\hline 0.3810 & $2018 / 09 / 26$ & 2.90 & 2.512 & 0.0630 & -2.8623 & -2.8123 & -2.7622 \\
\hline 0.3939 & $2018 / 12 / 26$ & 2.90 & 2.512 & 0.3123 & -1.2729 & -1.1615 & -1.0501 \\
\hline 0.4315 & $2018 / 09 / 26$ & 2.95 & 2.512 & 0.0630 & -3.2039 & -3.1538 & -3.1037 \\
\hline 0.4405 & $2018 / 12 / 26$ & 2.95 & 2.512 & 0.3123 & -1.4263 & -1.3149 & -1.2035 \\
\hline 0.4810 & $2018 / 09 / 26$ & 3.00 & 2.512 & 0.0630 & -3.5396 & -3.4896 & -3.4395 \\
\hline 0.5813 & $2018 / 09 / 26$ & 3.10 & 2.512 & 0.0630 & -4.1947 & -4.1447 & -4.0946 \\
\hline 0.6809 & $2018 / 09 / 26$ & 3.20 & 2.512 & 0.0630 & -4.8290 & -4.7789 & -4.7289 \\
\hline 0.7798 & $2018 / 09 / 26$ & 3.30 & 2.512 & 0.0630 & -5.4438 & -5.3937 & -5.3437 \\
\hline 0.8793 & $2018 / 09 / 26$ & 3.40 & 2.512 & 0.0630 & -6.0402 & -5.9901 & -5.9401 \\
\hline 0.9790 & $2018 / 09 / 26$ & 3.50 & 2.512 & 0.0630 & -6.6193 & -6.5692 & -6.5192 \\
\hline 1.0807 & $2018 / 09 / 26$ & 3.60 & 2.512 & 0.0630 & -7.1821 & -7.1320 & -7.0820 \\
\hline
\end{tabular}


4.2.1. Comparison of Pricing Effect on Call Options. There are 62 call options on September 4, 2018, and the current price of underlying asset $S_{0}=2.55$. Based on the estimated parameters $\mu=2.557 \%, \sigma=0.2005, r=1.768 \%$, $\lambda=0.0077963$ for call options, the call options of Shanghai 50 ETF on September 4, 2018, are priced by our obtained pricing formula in Theorem 3 and the Black-Scholes model, respectively; see Table 3 for detailed data, where $T$ is the expiration time of call option, $K$ is the strike price of call option, $S_{0}$ is the initial price of underlying asset, $\tau$ is the left expiration time of call option, $d_{1}, d_{2}$, and $d_{3}$ are the parameters in Theorem $3, C_{1}$ is the value of call option computed by the Black-Scholes model, $C_{2}$ is the value of call option computed by Theorem 3 , and $C$ is the actual closing price of call option.

According to simple computing, the expectation and variance of absolute errors follow as

$$
\begin{aligned}
& E\left(\left|\widehat{C_{1}}-C\right|\right)=0.0065574, \operatorname{Var}\left(\widehat{\mid C_{1}}-C \mid\right)=0.0045044, \\
& E\left(\mid \widehat{C_{2}-C \mid}\right)=0.0032696, \quad \operatorname{Var}\left(\widehat{\mid C_{2}}-C \mid\right)=0.001929
\end{aligned}
$$

where $C$ is the actual closing price of call option, $C_{1}$ is the value of call option computed by the Black-Scholes model, and $C_{2}$ is the value of call option computed by Theorem 3 . It is obvious that $E\left(\left|\widehat{C_{2}}-C\right|\right)<E\left(\left|\widehat{C_{1}-} C\right|\right)$. In the following, we will support the statement by the hypothesis test (i.e., $t$ test function of MATLAB R2018b).

In fact, for the hypothesis test,

$$
\begin{aligned}
& H_{0}: E\left(\left|C_{2}-C\right|\right)-E\left(\left|C_{1}-C\right|\right) \geq 0, \\
& H_{1}: E\left(\left|C_{2}-C\right|\right)-E\left(\left|C_{1}-C\right|\right)<0,
\end{aligned}
$$

The $t$-statistics equals -5.9311 with degree of freedom 61 , and its $p$ value is $7.6261 \times 10^{-8}$. Thus, we accept $H_{1}$, i.e., $E\left(\left|C_{2}-C\right|\right)<E\left(\left|C_{1}-C\right|\right)$. That is, the prices of call options computed by Theorem 3 are far nearer to their actual prices than those computed by the Black-Scholes model.

4.2.2. Comparison of Pricing Effect on Put Options. There are 62 put options on September 4, 2018, and the current price of underlying asset $S_{0}=2.55$. Based on the estimated parameters $\mu=2.557 \%, \sigma=0.2005, r=1.768 \%$, and $\lambda=0.013306$ for put options, the put options of Shanghai 50 ETF on September 4, 2018, are priced by our pricing formula in Theorem 2 and Black-Scholes model, respectively; see Table 4 for detailed data, where $T$ is the expiration time of put option, $K$ is the strike price of put option, $S_{0}$ is the initial price of underlying asset, $\tau$ is the left expiration time of put option, $d_{1}, d_{2}$, and $d_{3}$ are the parameters in Theorem $3, P_{1}$ is the value of put option computed by the Black-Scholes model, $P_{2}$ is the value of put option computed by Theorem 3 , and $P$ is the actual closing price of put option.

According to simple computing, the expectation and variance of absolute errors follow as

$$
\begin{aligned}
& E\left(\left|\widehat{P_{1}-P}\right|\right)=0.00836, \operatorname{Var}\left(\widehat{P_{1}}-P \mid\right)=0.0056946 \\
& E\left(\mid \widehat{P_{2}-P \mid}\right)=0.0044808, \operatorname{Var}\left(\widehat{\mid P_{2}}-P \mid\right)=0.0029812
\end{aligned}
$$

where $P$ is the actual closing price of put option, $P_{1}$ is the value of put option computed by the Black-Scholes model, and $P_{2}$ is the value of put option computed by Theorem 3 . It is obvious that $E\left(\left|\widehat{P_{2}}-P\right|\right)<E\left(\left|\widehat{P_{1}-P}\right|\right)$. In the following, we will support the statement by the hypothesis test (i.e., $t$ test function of MATLAB R2018b).

In fact, for the hypothesis test,

$$
\begin{aligned}
& H_{0}: E\left(\left|P_{2}-P\right|\right)-E\left(\left|P_{1}-P\right|\right) \geq 0, \\
& H_{1}: E\left(\left|P_{2}-P\right|\right)-E\left(\left|P_{1}-P\right|\right)<0,
\end{aligned}
$$

The $t$-statistics equals -4.7567 with degree of freedom 61 , and its $p$ value is $6.2304 \times 10^{-6}$. Thus, we accept $H_{1}$, i.e., $E\left(\left|P_{2}-P\right|\right)<E\left(\left|P_{1}-P\right|\right)$. That is, the prices of put options computed by Theorem 3 are far nearer to their actual prices than those computed by the Black-Scholes model.

Therefore, our pricing formulas in Theorem 2 and Theorem 3 have less absolute errors than those of the Black-Scholes model for both call options and put options. That is, the fitting effect of our pricing formulas is superior to that of the Black-Scholes model.

\section{Empirical Analysis of S\&P 500 Options}

In the section, we will present empirical analysis on S\&P 500 options and use the data of April 1 and 2, 2019 to compare the fitting effect of our pricing model and the Black-Scholes pricing model. All used data come from the Chicago Board Options Exchange, which includes actual option price $C$ or $P$, trading date $t$, exercise date $T$, strike price $K$, and current price of underlying asset $S_{0}$. We consider the out-of-money put and call options, which are more liquid and actively traded than in-the-money options. And observations with trading volume below average, prices less than $\$ 0.5$ or left expiration time less than 10 days or longer than 360 days are discarded. The annualized historical volatility $\sigma=0.1174$ based on closing prices of the underlying asset over the last month. According to the Board of Governors of the Federal Reserve System (https://www.federalreserve.gov/releases/ h15/data.htm), the annualized risk-free interest rate is 2.43\% on April 1 and 2.42\% on April 2, 2019.

5.1. Parameter Estimation. We consider the daily logarithmic returns of S\&P 500 index closing prices from January 2015 to December 2018. The augmented Dickey-Fuller test shows the Dickey-Fuller statistic equals -10.283 with lag order 10 and $p$ value is 0.01 , which indicates that the time series of returns is stationary. Furthermore, the autocorrelogram shows the coefficients of autocorrelation mostly fall within double standard deviations (see Figure 1). Thus, we accept that the time series of daily logarithmic returns of S\&P 500 index closing prices are stationary and independent. We take the annual average return rate based on 252 
TABLE 3: Pricing results by our pricing formula and the Black-Scholes model for call options.

\begin{tabular}{|c|c|c|c|c|c|c|c|c|c|}
\hline$T$ & K & $S_{0}$ & $\tau$ & $d_{1}$ & $d_{2}$ & $d_{3}$ & $C_{1}$ & $C_{2}$ & C \\
\hline $2018 / 09 / 26$ & 3.00 & 2.55 & 0.0603 & -3.2949 & -3.2457 & -3.1965 & 0.0005 & 0.0000 & 0.0005 \\
\hline $2018 / 12 / 26$ & 2.40 & 2.55 & 0.3096 & 0.5586 & 0.6702 & 0.7817 & 0.2239 & 0.2087 & 0.2200 \\
\hline $2018 / 12 / 26$ & 2.35 & 2.55 & 0.3096 & 0.7473 & 0.8589 & 0.9705 & 0.2601 & 0.2451 & 0.2555 \\
\hline 2018/09/26 & 2.90 & 2.55 & 0.0603 & -2.6062 & -2.5570 & -2.5077 & 0.0017 & 0.0002 & 0.0007 \\
\hline $2019 / 03 / 27$ & 2.50 & 2.55 & 0.5589 & 0.1525 & 0.3024 & 0.4523 & 0.2079 & 0.1883 & 0.2036 \\
\hline $2019 / 03 / 27$ & 2.55 & 2.55 & 0.5589 & 0.0204 & 0.1703 & 0.3202 & 0.1816 & 0.1626 & 0.1773 \\
\hline 2019/03/27 & 2.65 & 2.55 & 0.5589 & -0.2362 & -0.0863 & 0.0636 & 0.1364 & 0.1188 & 0.1353 \\
\hline $2018 / 12 / 26$ & 2.60 & 2.55 & 0.3096 & -0.1589 & -0.0473 & 0.0643 & 0.1107 & 0.0963 & 0.1067 \\
\hline 2018/09/26 & 2.20 & 2.55 & 0.0603 & 3.0060 & 3.0552 & 3.1044 & 0.3548 & 0.3520 & 0.3510 \\
\hline 2018/09/26 & 2.85 & 2.55 & 0.0603 & -2.2529 & -2.2036 & -2.1544 & 0.0030 & 0.0006 & 0.0012 \\
\hline $2019 / 03 / 27$ & 2.20 & 2.55 & 0.5589 & 1.0053 & 1.1552 & 1.3051 & 0.4160 & 0.3955 & 0.4070 \\
\hline $2018 / 12 / 26$ & 2.30 & 2.55 & 0.3096 & 0.9401 & 1.0517 & 1.1632 & 0.2991 & 0.2846 & 0.2925 \\
\hline $2018 / 10 / 24$ & 2.35 & 2.55 & 0.1370 & 1.1108 & 1.1850 & 1.2592 & 0.2264 & 0.2166 & 0.2255 \\
\hline $2018 / 10 / 24$ & 2.30 & 2.55 & 0.1370 & 1.4006 & 1.4748 & 1.5490 & 0.2698 & 0.2610 & 0.2653 \\
\hline $2018 / 10 / 24$ & 2.70 & 2.55 & 0.1370 & -0.7601 & -0.6859 & -0.6117 & 0.0356 & 0.0258 & 0.0300 \\
\hline $2018 / 12 / 26$ & 2.20 & 2.55 & 0.3096 & 1.3386 & 1.4501 & 1.5617 & 0.3840 & 0.3706 & 0.3817 \\
\hline 2018/09/26 & 2.50 & 2.55 & 0.0603 & 0.4090 & 0.4582 & 0.5074 & 0.0905 & 0.0800 & 0.0879 \\
\hline $2018 / 09 / 26$ & 2.95 & 2.55 & 0.0603 & -2.9535 & -2.9042 & -2.8550 & 0.0010 & 0.0001 & 0.0005 \\
\hline $2018 / 12 / 26$ & 2.95 & 2.55 & 0.3096 & -1.2909 & -1.1794 & -1.0678 & 0.0229 & 0.0150 & 0.0197 \\
\hline 2018/09/26 & 2.80 & 2.55 & 0.0603 & -1.8933 & -1.8441 & -1.7948 & 0.0052 & 0.0015 & 0.0019 \\
\hline $2018 / 12 / 26$ & 2.85 & 2.55 & 0.3096 & -0.9818 & -0.8703 & -0.7587 & 0.0371 & 0.0272 & 0.0341 \\
\hline $2018 / 10 / 24$ & 2.55 & 2.55 & 0.1370 & 0.0101 & 0.0843 & 0.1585 & 0.0901 & 0.0780 & 0.0854 \\
\hline $2018 / 10 / 24$ & 2.40 & 2.55 & 0.1370 & 0.8271 & 0.9013 & 0.9755 & 0.1862 & 0.1753 & 0.1837 \\
\hline 2018/09/26 & 3.10 & 2.55 & 0.0603 & -3.9610 & -3.9118 & -3.8626 & 0.0001 & 0.0000 & 0.0004 \\
\hline $2018 / 12 / 26$ & 2.70 & 2.55 & 0.3096 & -0.4972 & -0.3856 & -0.2740 & 0.0733 & 0.0604 & 0.0700 \\
\hline $2019 / 03 / 27$ & 2.35 & 2.55 & 0.5589 & 0.5653 & 0.7152 & 0.8651 & 0.3016 & 0.2811 & 0.2985 \\
\hline $2019 / 03 / 27$ & 2.40 & 2.55 & 0.5589 & 0.4249 & 0.5748 & 0.7246 & 0.2679 & 0.2476 & 0.2628 \\
\hline $2018 / 12 / 26$ & 2.75 & 2.55 & 0.3096 & -0.6616 & -0.5501 & -0.4385 & 0.0588 & 0.0469 & 0.0550 \\
\hline $2019 / 03 / 27$ & 2.25 & 2.55 & 0.5589 & 0.8554 & 1.0053 & 1.1552 & 0.3758 & 0.3552 & 0.3679 \\
\hline $2019 / 03 / 27$ & 2.80 & 2.55 & 0.5589 & -0.6035 & -0.4536 & -0.3038 & 0.0856 & 0.0707 & 0.0810 \\
\hline $2018 / 12 / 26$ & 2.65 & 2.55 & 0.3096 & -0.3296 & -0.2181 & -0.1065 & 0.0905 & 0.0768 & 0.0871 \\
\hline $2018 / 09 / 26$ & 2.30 & 2.55 & 0.0603 & 2.1029 & 2.1521 & 2.2014 & 0.2571 & 0.2528 & 0.2538 \\
\hline $2018 / 09 / 26$ & 3.60 & 2.55 & 0.0603 & -6.9988 & -6.9496 & -6.9003 & 0.0000 & 0.0000 & 0.0004 \\
\hline $2019 / 03 / 27$ & 2.75 & 2.55 & 0.5589 & -0.4833 & -0.3334 & -0.1835 & 0.1004 & 0.0846 & 0.0988 \\
\hline $2019 / 03 / 27$ & 2.30 & 2.55 & 0.5589 & 0.7088 & 0.8587 & 1.0086 & 0.3376 & 0.3170 & 0.3316 \\
\hline $2018 / 09 / 26$ & 2.40 & 2.55 & 0.0603 & 1.2383 & 1.2875 & 1.3368 & 0.1658 & 0.1583 & 0.1641 \\
\hline $2018 / 09 / 26$ & 2.25 & 2.55 & 0.0603 & 2.5494 & 2.5986 & 2.6479 & 0.3055 & 0.3022 & 0.3040 \\
\hline $2019 / 03 / 27$ & 2.60 & 2.55 & 0.5589 & -0.1091 & 0.0408 & 0.1907 & 0.1578 & 0.1394 & 0.1549 \\
\hline $2018 / 10 / 24$ & 2.45 & 2.55 & 0.1370 & 0.5492 & 0.6234 & 0.6976 & 0.1497 & 0.1380 & 0.1461 \\
\hline $2018 / 12 / 26$ & 2.90 & 2.55 & 0.3096 & -1.1377 & -1.0262 & -0.9146 & 0.0292 & 0.0203 & 0.0266 \\
\hline $2018 / 10 / 24$ & 2.50 & 2.55 & 0.1370 & 0.2770 & 0.3512 & 0.4254 & 0.1175 & 0.1054 & 0.1135 \\
\hline $2018 / 10 / 24$ & 2.75 & 2.55 & 0.1370 & -1.0074 & -0.9332 & -0.8590 & 0.0252 & 0.0167 & 0.0195 \\
\hline $2018 / 09 / 26$ & 3.30 & 2.55 & 0.0603 & -5.2311 & -5.1819 & -5.1327 & 0.0000 & 0.0000 & 0.0003 \\
\hline $2018 / 12 / 26$ & 2.50 & 2.55 & 0.3096 & 0.1927 & 0.3043 & 0.4158 & 0.1607 & 0.1455 & 0.1572 \\
\hline $2018 / 12 / 26$ & 2.45 & 2.55 & 0.3096 & 0.3738 & 0.4854 & 0.5969 & 0.1907 & 0.1754 & 0.1870 \\
\hline $2018 / 10 / 24$ & 2.60 & 2.55 & 0.1370 & -0.2516 & -0.1774 & -0.1031 & 0.0675 & 0.0559 & 0.0623 \\
\hline 2018/09/26 & 2.70 & 2.55 & 0.0603 & -1.1545 & -1.1053 & -1.0560 & 0.0153 & 0.0081 & 0.0103 \\
\hline $2018 / 12 / 26$ & 2.80 & 2.55 & 0.3096 & -0.8232 & -0.7116 & -0.6000 & 0.0469 & 0.0359 & 0.0445 \\
\hline 2018/09/26 & 2.55 & 2.55 & 0.0603 & 0.0067 & 0.0559 & 0.1052 & 0.0621 & 0.0512 & 0.0590 \\
\hline $2018 / 09 / 26$ & 3.50 & 2.55 & 0.0603 & -6.4265 & -6.3773 & -6.3280 & 0.0000 & 0.0000 & 0.0003 \\
\hline $2018 / 09 / 26$ & 2.65 & 2.55 & 0.0603 & -0.7747 & -0.7255 & -0.6763 & 0.0253 & 0.0164 & 0.0206 \\
\hline 2018/09/26 & 2.35 & 2.55 & 0.0603 & 1.6660 & 1.7152 & 1.7645 & 0.2101 & 0.2045 & 0.2077 \\
\hline $2018 / 09 / 26$ & 2.45 & 2.55 & 0.0603 & 0.8194 & 0.8686 & 0.9179 & 0.1254 & 0.1162 & 0.1232 \\
\hline $2018 / 12 / 26$ & 2.25 & 2.55 & 0.3096 & 1.1371 & 1.2487 & 1.3602 & 0.3405 & 0.3265 & 0.3330 \\
\hline $2018 / 10 / 24$ & 2.65 & 2.55 & 0.1370 & -0.5083 & -0.4340 & -0.3598 & 0.0495 & 0.0387 & 0.0442 \\
\hline $2018 / 09 / 26$ & 2.75 & 2.55 & 0.0603 & -1.5272 & -1.4780 & -1.4288 & 0.0090 & 0.0037 & 0.0048 \\
\hline $2018 / 09 / 26$ & 3.40 & 2.55 & 0.0603 & -5.8376 & -5.7884 & -5.7392 & 0.0000 & 0.0000 & 0.0003 \\
\hline $2019 / 03 / 27$ & 2.70 & 2.55 & 0.5589 & -0.3609 & -0.2110 & -0.0611 & 0.1173 & 0.1006 & 0.1162 \\
\hline $2018 / 09 / 26$ & 3.20 & 2.55 & 0.0603 & -4.6060 & -4.5568 & -4.5076 & 0.0000 & 0.0000 & 0.0003 \\
\hline $2018 / 12 / 26$ & 2.55 & 2.55 & 0.3096 & 0.0152 & 0.1268 & 0.2383 & 0.1341 & 0.1192 & 0.1311 \\
\hline 2018/09/26 & 2.60 & 2.55 & 0.0603 & -0.3878 & -0.3386 & -0.2893 & 0.0405 & 0.0303 & 0.0377 \\
\hline $2019 / 03 / 27$ & 2.45 & 2.55 & 0.5589 & 0.2873 & 0.4372 & 0.5871 & 0.2367 & 0.2166 & 0.2319 \\
\hline
\end{tabular}


TABLE 4: Pricing results by our pricing formula and the Black-Scholes model for put options.

\begin{tabular}{|c|c|c|c|c|c|c|c|c|c|}
\hline$T$ & $K$ & $S_{0}$ & $\tau$ & $d_{1}$ & $d_{2}$ & $d_{3}$ & $P_{1}$ & $P_{2}$ & $P$ \\
\hline $2018 / 12 / 26$ & 2.50 & 2.55 & 0.3096 & 0.1927 & 0.3043 & 0.4158 & 0.0963 & 0.0839 & 0.0983 \\
\hline 2019/03/27 & 2.30 & 2.55 & 0.5589 & 0.7088 & 0.8587 & 1.0086 & 0.0583 & 0.0478 & 0.0635 \\
\hline $2019 / 03 / 27$ & 2.25 & 2.55 & 0.5589 & 0.8554 & 1.0053 & 1.1552 & 0.0463 & 0.0364 & 0.0520 \\
\hline $2018 / 12 / 26$ & 2.20 & 2.55 & 0.3096 & 1.3386 & 1.4501 & 1.5617 & 0.0186 & 0.0104 & 0.0205 \\
\hline $2018 / 12 / 26$ & 2.90 & 2.55 & 0.3096 & -1.1377 & -1.0262 & -0.9146 & 0.3631 & 0.3568 & 0.3618 \\
\hline 2018/09/26 & 2.30 & 2.55 & 0.0603 & 2.1029 & 2.1521 & 2.2014 & 0.0050 & 0.0007 & 0.0030 \\
\hline 2018/09/26 & 3.20 & 2.55 & 0.0603 & -4.6060 & -4.5568 & -4.5076 & 0.6473 & 0.6471 & 0.6437 \\
\hline $2018 / 10 / 24$ & 2.70 & 2.55 & 0.1370 & v0.7601 & -0.6859 & -0.6117 & 0.1831 & 0.1703 & 0.1724 \\
\hline 2018/09/26 & 2.50 & 2.55 & 0.0603 & 0.4090 & 0.4582 & 0.5074 & 0.0440 & 0.0278 & 0.0359 \\
\hline 2018/09/26 & 2.65 & 2.55 & 0.0603 & -0.7747 & -0.7255 & -0.6763 & 0.1282 & 0.1140 & 0.1191 \\
\hline $2019 / 03 / 27$ & 2.55 & 2.55 & 0.5589 & 0.0204 & 0.1703 & 0.3202 & 0.1521 & 0.1413 & 0.1569 \\
\hline $2018 / 10 / 24$ & 2.60 & 2.55 & 0.1370 & -0.2516 & -0.1774 & -0.1031 & 0.1162 & 0.1005 & 0.1054 \\
\hline 2018/09/26 & 2.20 & 2.55 & 0.0603 & 3.0060 & 3.0552 & 3.1044 & 0.0012 & 0.0000 & 0.0010 \\
\hline $2019 / 03 / 27$ & 2.40 & 2.55 & 0.5589 & 0.4249 & 0.5748 & 0.7246 & 0.0887 & 0.0775 & 0.0939 \\
\hline $2019 / 03 / 27$ & 2.60 & 2.55 & 0.5589 & -0.1091 & 0.0408 & 0.1907 & 0.1780 & 0.1677 & 0.1829 \\
\hline 2018/09/26 & 2.85 & 2.55 & 0.0603 & -2.2529 & -2.2036 & -2.1544 & 0.3010 & 0.2980 & 0.2955 \\
\hline $2018 / 12 / 26$ & 2.40 & 2.55 & 0.3096 & 0.5586 & 0.6702 & 0.7817 & 0.0605 & 0.0475 & 0.0607 \\
\hline $2019 / 03 / 27$ & 2.45 & 2.55 & 0.5589 & 0.2873 & 0.4372 & 0.5871 & 0.1074 & 0.0962 & 0.1121 \\
\hline $2019 / 03 / 27$ & 2.65 & 2.55 & 0.5589 & -0.2362 & -0.0863 & 0.0636 & 0.2062 & 0.1967 & 0.2094 \\
\hline $2019 / 03 / 27$ & 2.80 & 2.55 & 0.5589 & -0.6035 & -0.4536 & -0.3038 & 0.3040 & 0.2973 & 0.3078 \\
\hline $2018 / 09 / 26$ & 2.45 & 2.55 & 0.0603 & 0.8194 & 0.8686 & 0.9179 & 0.0277 & 0.0140 & 0.0219 \\
\hline $2018 / 12 / 26$ & 2.95 & 2.55 & 0.3096 & -1.2909 & -1.1794 & -1.0678 & 0.4061 & 0.4013 & 0.4056 \\
\hline $2018 / 12 / 26$ & 2.25 & 2.55 & 0.3096 & 1.1371 & 1.2487 & 1.3602 & 0.0257 & 0.0161 & 0.0271 \\
\hline 2018/09/26 & 2.80 & 2.55 & 0.0603 & -1.8933 & -1.8441 & -1.7948 & 0.2541 & 0.2490 & 0.2486 \\
\hline $2018 / 10 / 24$ & 2.65 & 2.55 & 0.1370 & -0.5083 & -0.4340 & -0.3598 & 0.1478 & 0.1332 & 0.1339 \\
\hline $2018 / 12 / 26$ & 2.75 & 2.55 & 0.3096 & -0.6616 & -0.5501 & -0.4385 & 0.2446 & 0.2341 & 0.2387 \\
\hline $2018 / 12 / 26$ & 2.85 & 2.55 & 0.3096 & -0.9818 & -0.8703 & -0.7587 & 0.3217 & 0.3140 & 0.3201 \\
\hline 2018/09/26 & 2.70 & 2.55 & 0.0603 & -1.1545 & -1.1053 & -1.0560 & 0.1668 & 0.1557 & 0.1585 \\
\hline 2018/09/26 & 3.50 & 2.55 & 0.0603 & -6.4265 & -6.3773 & -6.3280 & 0.9470 & 0.9468 & 0.9443 \\
\hline $2019 / 03 / 27$ & 2.35 & 2.55 & 0.5589 & 0.5653 & 0.7152 & 0.8651 & 0.0724 & 0.0614 & 0.0784 \\
\hline $2018 / 10 / 24$ & 2.55 & 2.55 & 0.1370 & 0.0101 & 0.0843 & 0.1585 & 0.0889 & 0.0728 & 0.0788 \\
\hline $2018 / 12 / 26$ & 2.70 & 2.55 & 0.3096 & -0.4972 & -0.3856 & -0.2740 & 0.2096 & 0.1979 & 0.2079 \\
\hline $2018 / 10 / 24$ & 2.75 & 2.55 & 0.1370 & -1.0074 & -0.9332 & -0.8590 & 0.2219 & 0.2110 & 0.2120 \\
\hline $2018 / 12 / 26$ & 2.45 & 2.55 & 0.3096 & 0.3738 & 0.4854 & 0.5969 & 0.0776 & 0.0640 & 0.0782 \\
\hline $2018 / 10 / 24$ & 2.40 & 2.55 & 0.1370 & 0.8271 & 0.9013 & 0.9755 & 0.0333 & 0.0204 & 0.0260 \\
\hline 2018/09/26 & 3.10 & 2.55 & 0.0603 & -3.9610 & -3.9118 & -3.8626 & 0.5474 & 0.5472 & 0.5451 \\
\hline $2018 / 10 / 24$ & 2.50 & 2.55 & 0.1370 & 0.2770 & 0.3512 & 0.4254 & 0.0661 & 0.0503 & 0.0569 \\
\hline $2018 / 09 / 26$ & 3.30 & 2.55 & 0.0603 & -5.2311 & -5.1819 & -5.1327 & 0.7472 & 0.7470 & 0.7420 \\
\hline $2018 / 10 / 24$ & 2.30 & 2.55 & 0.1370 & 1.4006 & 1.4748 & 1.5490 & 0.0149 & 0.0063 & 0.0108 \\
\hline $2018 / 12 / 26$ & 2.35 & 2.55 & 0.3096 & 0.7473 & 0.8589 & 0.9705 & 0.0463 & 0.0343 & 0.0467 \\
\hline $2018 / 09 / 26$ & 2.40 & 2.55 & 0.0603 & 1.2383 & 1.2875 & 1.3368 & 0.0166 & 0.0062 & 0.0118 \\
\hline 2018/09/26 & 3.40 & 2.55 & 0.0603 & -5.8376 & -5.7884 & -5.7392 & 0.8471 & 0.8469 & 0.8453 \\
\hline $2018 / 09 / 26$ & 2.60 & 2.55 & 0.0603 & -0.3878 & -0.3386 & -0.2893 & 0.0943 & 0.0779 & 0.0844 \\
\hline 2018/09/26 & 2.25 & 2.55 & 0.0603 & 2.5494 & 2.5986 & 2.6479 & 0.0025 & 0.0002 & 0.0015 \\
\hline $2018 / 10 / 24$ & 2.35 & 2.55 & 0.1370 & 1.1108 & 1.1850 & 1.2592 & 0.0226 & 0.0118 & 0.0178 \\
\hline $2019 / 03 / 27$ & 2.70 & 2.55 & 0.5589 & -0.3609 & -0.2110 & -0.0611 & 0.2368 & 0.2280 & 0.2402 \\
\hline $2018 / 09 / 26$ & 2.35 & 2.55 & 0.0603 & 1.6660 & 1.7152 & 1.7645 & 0.0094 & 0.0023 & 0.0065 \\
\hline 2018/09/26 & 2.95 & 2.55 & 0.0603 & -2.9535 & -2.9042 & -2.8550 & 0.3982 & 0.3974 & 0.3921 \\
\hline $2018 / 10 / 24$ & 2.45 & 2.55 & 0.1370 & 0.5492 & 0.6234 & 0.6976 & 0.0476 & 0.0330 & 0.0387 \\
\hline $2018 / 12 / 26$ & 2.65 & 2.55 & 0.3096 & -0.3296 & -0.2181 & -0.1065 & 0.1772 & 0.1645 & 0.1753 \\
\hline $2018 / 12 / 26$ & 2.55 & 2.55 & 0.3096 & 0.0152 & 0.1268 & 0.2383 & 0.1212 & 0.1073 & 0.1210 \\
\hline $2019 / 03 / 27$ & 2.75 & 2.55 & 0.5589 & -0.4833 & -0.3334 & -0.1835 & 0.2694 & 0.2616 & 0.2738 \\
\hline 2018/09/26 & 2.75 & 2.55 & 0.0603 & -1.5272 & -1.4780 & -1.4288 & 0.2091 & 0.2012 & 0.2011 \\
\hline $2018 / 09 / 26$ & 3.60 & 2.55 & 0.0603 & -6.9988 & -6.9496 & -6.9003 & 1.0469 & 1.0467 & 1.0452 \\
\hline $2019 / 03 / 27$ & 2.20 & 2.55 & 0.5589 & 1.0053 & 1.1552 & 1.3051 & 0.0363 & 0.0272 & 0.0423 \\
\hline $2018 / 12 / 26$ & 2.80 & 2.55 & 0.3096 & -0.8232 & -0.7116 & -0.6000 & 0.2821 & 0.2729 & 0.2800 \\
\hline $2018 / 12 / 26$ & 2.30 & 2.55 & 0.3096 & 0.9401 & 1.0517 & 1.1632 & 0.0348 & 0.0239 & 0.0353 \\
\hline 2018/09/26 & 2.90 & 2.55 & 0.0603 & -2.6062 & -2.5570 & -2.5077 & 0.3492 & 0.3476 & 0.3430 \\
\hline $2018 / 09 / 26$ & 2.55 & 2.55 & 0.0603 & 0.0067 & 0.0559 & 0.1052 & 0.0661 & 0.0489 & 0.0573 \\
\hline $2019 / 03 / 27$ & 2.50 & 2.55 & 0.5589 & 0.1525 & 0.3024 & 0.4523 & 0.1285 & 0.1174 & 0.1330 \\
\hline $2018 / 12 / 26$ & 2.60 & 2.55 & 0.3096 & -0.1589 & -0.0473 & 0.0643 & 0.1477 & 0.1342 & 0.1468 \\
\hline $2018 / 09 / 26$ & 3.00 & 2.55 & 0.0603 & -3.2949 & -3.2457 & -3.1965 & 0.4477 & 0.4473 & 0.4459 \\
\hline
\end{tabular}




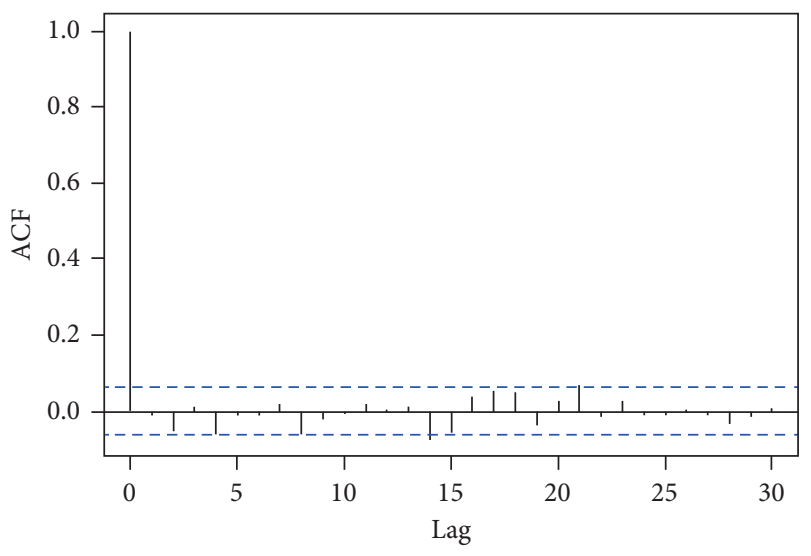

FIGURE 1: The autocorrelogram of the daily logarithmic returns of S\&P 500 index closing prices.

effective trading days as the drift coefficient of the underlying asset $\mu$.

There are 41 call options on April 1, 2019, and the current price of underlying asset $S_{0}=2867.19$. According to simple computation, we obtain $\mu=4.932 \%$ and the left expiration time $\tau=(T-t) / 365$ (years), where $t=2019 / 04 / 01$, and then we work out $d_{1}, d_{2}$, and $d_{3}$ by Theorem 2 ; see Table 5 for detailed data, where $C$ is the actual closing price of call option, $\tau$ is the left expiration time of call option, $K$ is the strike price of call option, and $d_{1}, d_{2}$, and $d_{3}$ are the parameters in Theorem 2. Furthermore, we obtain the estimated value of the risk-compensation factor $\lambda=-0.0246533$ by the least square method with $R^{2}=0.9532$.

There are 62 put options on April 1, 2019, and the current price of underlying asset $S_{0}=2867.19$. Similarly, we obtain $\mu=4.932 \%$ and the left expiration time $\tau=(T-t) / 365$ (years), where $t=2019 / 04 / 01$, and then we work out $d_{1}, d_{2}$, and $d_{3}$ by Theorem 3 ; see Table 6 for detailed data, where $P$ is the actual closing price of put option, $\tau$ is the left expiration time of put option, $K$ is the strike price of put option, and $d_{1}, d_{2}$, and $d_{3}$ are the parameters in Theorem 3 . Furthermore, we obtain the estimated value of the riskcompensation factor $\lambda=0.0269767$ by the least square method with $R^{2}=0.9561$.

5.2. Comparison of Pricing Effect. In the section, we will compare the fitting effect of our pricing formulas with the Black-Scholes model.

5.2.1. Comparison of Pricing Effect on Call Options. There are 25 call options on April 2, 2019, and the current price of underlying asset $S_{0}=2867.24$. Based on the estimated parameters $\mu=4.932 \%, \sigma=0.1174, r=2.42 \%$, and $\lambda=-0.0246533$ for call options, the call options of S\&P 500 on April 2, 2019, are priced by our obtained pricing formula in Theorem 1 and the Black-Scholes model, respectively; see Table 7 for detailed data, where $K$ is the strike price of call option, $\tau$ is the left expiration time of call option, $d_{1}, d_{2}$, and $d_{3}$ are the parameters in Theorem 3, C is the actual closing price of call option, $C_{1}$ is the value of call option computed by the Black-Scholes model, and $C_{2}$ is the value of call option computed by Theorem 1 .

According to simple computing, the expectation and variance of absolute errors follow as

$$
\begin{aligned}
& E\left(\left|\widehat{C_{1}}-C\right|\right)=6.902386, \operatorname{Var}\left(\widehat{\mid C_{1}}-C \mid\right)=6.810772, \\
& E\left(\left|\widehat{C_{2}}-C\right|\right)=5.156575, \operatorname{Var}\left(\widehat{\mid C_{2}}-C \mid\right)=9.726556,
\end{aligned}
$$

where $C$ is the actual closing price of call option, $C_{1}$ is the value of call option computed by the Black-Scholes model, and $C_{2}$ is the value of call option computed by Theorem 2 . It is obvious that $E\left(\left|\widehat{C_{2}}-C\right|\right)<E\left(\left|\widehat{C_{1}-} C\right|\right)$. In the following, we will support the statement by the hypothesis test (i.e., test function of MATLAB R2018b).

In fact, for the hypothesis test,

$$
\begin{aligned}
& H_{0}: E\left(\left|C_{2}-C\right|\right)-E\left(\left|C_{1}-C\right|\right) \geq 0, \\
& H_{1}: E\left(\left|C_{2}-C\right|\right)-E\left(\left|C_{1}-C\right|\right)<0,
\end{aligned}
$$

the $t$-statistics equals -2.1465 with degree of freedom 24 , and its $p$ value is 0.01854 . Thus, we accept $H_{1}$, i.e., $E\left(\left|C_{2}-C\right|\right)<E\left(\left|C_{1}-C\right|\right)$. That is, the prices of call options computed by Theorem 2 are far nearer to their actual prices than those computed by the Black-Scholes model.

5.2.2. Comparison of Pricing Effect on Put Options. There are 39 put options on April 2, 2019, and the current price of underlying asset $S_{0}=2867.24$. Based on the estimated parameters $\mu=4.932 \%, \sigma=0.1174, \quad r=2.42 \%$, and $\lambda=$ 0.0269767 for put options, the put options of S\&P 500 on April 2, 2019, are priced by our obtained pricing formula in Theorem 3 and the Black-Scholes model, respectively; see Table 8 for detailed data, where $K$ is the strike price of put option, $\tau$ is the left expiration time of put option, $d_{1}, d_{2}$, and $d_{3}$ are the parameters in Theorem 3, $P$ is the actual closing price of put option, $P_{1}$ is the value of put option computed by the Black-Scholes model, and $P_{2}$ is the value of put option computed by Theorem 3 .

According to simple computing, the expectation and variance of absolute errors follow as

$$
\begin{aligned}
& E\left(\left|\widehat{P_{1}-P}\right|\right)=7.850124, \operatorname{Var}\left(\widehat{\mid P_{1}}-P \mid\right)=29.945065, \\
& E\left(\left|\widehat{P_{2}-P}\right|\right)=3.492115, \operatorname{Var}\left(\widehat{P_{2}}-P \mid\right)=6.764690,
\end{aligned}
$$

where $P$ is the actual closing price of put option, $P_{1}$ is the value of put option computed by the Black-Scholes model, and $P_{2}$ is the value of put option computed by Theorem 3. It is obvious that $E\left(\left|\widehat{P_{2}}-P\right|\right)<E\left(\left|\widehat{P_{1}}-P\right|\right)$. In the following, we will support the statement by the hypothesis test (i.e., $t$ test function of MATLAB R2018b).

In fact, for the hypothesis test,

$$
\begin{aligned}
& H_{0}: E\left(\left|P_{2}-P\right|\right)-E\left(\left|P_{1}-P\right|\right) \geq 0, \\
& H_{1}: E\left(\left|P_{2}-P\right|\right)-E\left(\left|P_{1}-P\right|\right)<0,
\end{aligned}
$$


TABle 5: Parameter estimation of $d_{1}, d_{2}$, and $d_{3}$ for call options.

\begin{tabular}{|c|c|c|c|c|c|}
\hline$C$ & $\tau$ & $K$ & $d_{1}$ & $d_{2}$ & $d_{3}$ \\
\hline 24.40 & 0.046575 & 2870 & 0.039320 & 0.064659 & 0.089997 \\
\hline 21.50 & 0.046575 & 2875 & -0.029375 & -0.004037 & 0.021302 \\
\hline 10.60 & 0.046575 & 2900 & -0.371070 & -0.345732 & -0.320393 \\
\hline 4.50 & 0.046575 & 2925 & -0.709832 & -0.684493 & -0.659155 \\
\hline 3.85 & 0.046575 & 2930 & -0.777237 & -0.751898 & -0.726559 \\
\hline 3.14 & 0.046575 & 2935 & -0.844526 & -0.819188 & -0.793849 \\
\hline 1.67 & 0.046575 & 2950 & -1.045710 & -1.020372 & -0.995033 \\
\hline 0.65 & 0.046575 & 2975 & -1.378754 & -1.353416 & -1.328077 \\
\hline 0.60 & 0.046575 & 2980 & -1.445027 & -1.419689 & -1.394350 \\
\hline 46.15 & 0.126027 & 2870 & 0.104771 & 0.146452 & 0.188133 \\
\hline 44.00 & 0.126027 & 2875 & 0.063010 & 0.104691 & 0.146371 \\
\hline 31.00 & 0.126027 & 2900 & -0.144713 & -0.103032 & -0.061351 \\
\hline 19.35 & 0.126027 & 2925 & -0.350653 & -0.308972 & -0.267291 \\
\hline 7.00 & 0.126027 & 2975 & -0.757303 & -0.715622 & -0.673941 \\
\hline 3.73 & 0.126027 & 3000 & -0.958073 & -0.916392 & -0.874711 \\
\hline 3.30 & 0.126027 & 3005 & -0.998026 & -0.956345 & -0.914664 \\
\hline 2.50 & 0.126027 & 3015 & -1.077733 & -1.036052 & -0.994371 \\
\hline 66.70 & 0.221918 & 2870 & 0.152504 & 0.207814 & 0.263123 \\
\hline 63.80 & 0.221918 & 2875 & 0.121033 & 0.176343 & 0.231652 \\
\hline 48.60 & 0.221918 & 2900 & -0.035505 & 0.019805 & 0.075114 \\
\hline 36.70 & 0.221918 & 2925 & -0.190699 & -0.135390 & -0.080080 \\
\hline 28.32 & 0.221918 & 2940 & -0.283181 & -0.227871 & -0.172561 \\
\hline 27.70 & 0.221918 & 2950 & -0.344573 & -0.289263 & -0.233954 \\
\hline 13.03 & 0.221918 & 3000 & -0.648446 & -0.593137 & -0.537827 \\
\hline 6.00 & 0.221918 & 3050 & -0.947297 & -0.891987 & -0.836678 \\
\hline 3.10 & 0.221918 & 3100 & -1.241288 & -1.185978 & -1.130669 \\
\hline 0.85 & 0.221918 & 3200 & -1.815306 & -1.759996 & -1.704686 \\
\hline 28.30 & 0.298630 & 2975 & -0.377841 & -0.313680 & -0.249519 \\
\hline 21.90 & 0.298630 & 3000 & -0.508267 & -0.444106 & -0.379945 \\
\hline 17.00 & 0.375342 & 3050 & -0.637911 & -0.565980 & -0.494048 \\
\hline 42.20 & 0.471233 & 3000 & -0.313762 & -0.233165 & -0.152567 \\
\hline 15.10 & 0.471233 & 3100 & -0.720596 & -0.639998 & -0.559401 \\
\hline 133.50 & 0.720548 & 2875 & 0.279420 & 0.379083 & 0.478746 \\
\hline 69.70 & 0.720548 & 3000 & -0.147614 & -0.047950 & 0.051713 \\
\hline 33.36 & 0.720548 & 3100 & -0.476619 & -0.376956 & -0.277292 \\
\hline 27.50 & 0.720548 & 3125 & -0.557212 & -0.457549 & -0.357885 \\
\hline 15.70 & 0.720548 & 3200 & -0.795178 & -0.695515 & -0.595852 \\
\hline 7.00 & 0.720548 & 3300 & -1.103934 & -1.004271 & -0.904607 \\
\hline 75.30 & 0.797260 & 3000 & -0.109289 & -0.004455 & 0.100380 \\
\hline 18.70 & 0.797260 & 3200 & -0.724912 & -0.620077 & -0.515243 \\
\hline 52.44 & 0.969863 & 3100 & -0.319343 & -0.203716 & -0.088089 \\
\hline
\end{tabular}

TABLe 6: Parameter estimation of $d_{1}, d_{2}$, and $d_{3}$ for put options.

\begin{tabular}{lccccc}
\hline$P$ & $\tau$ & $K$ & $d_{1}$ & $d_{2}$ & $d_{3}$ \\
\hline 0.35 & 0.046575 & 2400 & 7.097458 & 7.122797 & 7.148136 \\
0.42 & 0.046575 & 2410 & 6.933361 & 6.958699 & 6.795281 \\
0.40 & 0.046575 & 2420 & 6.769942 & 6.551415 & 6.820619 \\
0.40 & 0.046575 & 2435 & 6.526076 & 6.470460 & 6.576753 \\
0.47 & 2440 & 6.445121 & 6.309047 & 6.495799 \\
0.46 & 0.046575 & 2450 & 6.283708 & 6.228587 & 6.334385 \\
0.55 & 0.046575 & 2455 & 6.203248 & 5.749243 & 5.659915 \\
0.54 & 0.046575 & 2485 & 5.723905 & 5.511737 & 5.695254 \\
0.62 & 0.046575 & 2490 & 5.644577 & 5.275652 & 5.537076 \\
0.56 & 0.046575 & 2500 & 5.486398 & 5.119042 & 5.300990 \\
0.72 & 0.046575 & 2515 & 5.093704 & 4.730217 & 5.144381 \\
0.74 & 0.046575 & 2525 & 4.704878 & 3.963872 & 3.9895211 \\
0.75 & 0.046575 & 2550 & 3.938534 & & \\
0.80 & 0.046575 & 2600 & &
\end{tabular}


TABle 6: Continued.

\begin{tabular}{|c|c|c|c|c|c|}
\hline$P$ & $\tau$ & $K$ & $d_{1}$ & $d_{2}$ & $d_{3}$ \\
\hline 0.93 & 0.046575 & 2605 & 3.862711 & 3.888050 & 3.913389 \\
\hline 1.38 & 0.046575 & 2640 & 3.335995 & 3.361334 & 3.386673 \\
\hline 1.50 & 0.046575 & 2650 & 3.186787 & 3.212126 & 3.237464 \\
\hline 1.80 & 0.046575 & 2675 & 2.816217 & 2.841555 & 2.866894 \\
\hline 2.44 & 0.046575 & 2700 & 2.449093 & 2.474432 & 2.499770 \\
\hline 2.55 & 0.046575 & 2705 & 2.376076 & 2.401415 & 2.426754 \\
\hline 2.65 & 0.046575 & 2710 & 2.303195 & 2.328533 & 2.353872 \\
\hline 3.03 & 0.046575 & 2720 & 2.157833 & 2.183172 & 2.208511 \\
\hline 3.25 & 0.046575 & 2725 & 2.085353 & 2.110692 & 2.136030 \\
\hline 4.50 & 0.046575 & 2750 & 1.724935 & 1.750274 & 1.775612 \\
\hline 0.55 & 0.126027 & 2000 & 8.769770 & 8.811451 & 8.853132 \\
\hline 1.15 & 0.126027 & 2275 & 5.678836 & 5.720517 & 5.762198 \\
\hline 1.50 & 0.126027 & 2350 & 4.900655 & 4.942336 & 4.984017 \\
\hline 1.95 & 0.126027 & 2400 & 4.395546 & 4.437227 & 4.478907 \\
\hline 2.20 & 0.126027 & 2425 & 4.146924 & 4.188605 & 4.230285 \\
\hline 3.20 & 0.126027 & 2500 & 3.416152 & 3.457833 & 3.499514 \\
\hline 5.10 & 0.126027 & 2575 & 2.706983 & 2.748664 & 2.790345 \\
\hline 6.05 & 0.126027 & 2600 & 2.475176 & 2.516857 & 2.558538 \\
\hline 6.38 & 0.126027 & 2610 & 2.383077 & 2.424758 & 2.466439 \\
\hline 8.55 & 0.126027 & 2650 & 2.018175 & 2.059856 & 2.101537 \\
\hline 10.31 & 0.126027 & 2675 & 1.792898 & 1.834579 & 1.876260 \\
\hline 12.35 & 0.126027 & 2700 & 1.569717 & 1.611398 & 1.653079 \\
\hline 14.35 & 0.126027 & 2725 & 1.348593 & 1.390274 & 1.431955 \\
\hline 18.07 & 0.126027 & 2750 & 1.129488 & 1.171169 & 1.212850 \\
\hline 22.54 & 0.126027 & 2775 & 0.912366 & 0.954047 & 0.995728 \\
\hline 27.70 & 0.126027 & 2800 & 0.697191 & 0.738872 & 0.780553 \\
\hline 32.85 & 0.126027 & 2825 & 0.483929 & 0.525610 & 0.567291 \\
\hline 41.25 & 0.126027 & 2850 & 0.272547 & 0.314227 & 0.355908 \\
\hline 1.30 & 0.221918 & 2000 & 6.682380 & 6.737690 & 6.793000 \\
\hline 5.39 & 0.221918 & 2375 & 3.575320 & 3.630630 & 3.685939 \\
\hline 5.80 & 0.221918 & 2400 & 3.385999 & 3.441308 & 3.496618 \\
\hline 9.26 & 0.221918 & 2500 & 2.647935 & 2.703245 & 2.758554 \\
\hline 11.74 & 0.221918 & 2550 & 2.289903 & 2.345212 & 2.400522 \\
\hline 12.90 & 0.221918 & 2565 & 2.183861 & 2.239171 & 2.294480 \\
\hline 13.55 & 0.221918 & 2575 & 2.113511 & 2.168820 & 2.224130 \\
\hline 15.32 & 0.221918 & 2600 & 1.938823 & 1.994133 & 2.049442 \\
\hline 17.70 & 0.221918 & 2625 & 1.765807 & 1.821117 & 1.876426 \\
\hline 19.60 & 0.221918 & 2650 & 1.594431 & 1.649740 & 1.705050 \\
\hline 26.23 & 0.221918 & 2700 & 1.256476 & 1.311786 & 1.367095 \\
\hline 28.70 & 0.221918 & 2715 & 1.156310 & 1.211619 & 1.266929 \\
\hline 31.25 & 0.221918 & 2725 & 1.089839 & 1.145148 & 1.200458 \\
\hline 35.95 & 0.221918 & 2750 & 0.924723 & 0.980033 & 1.035342 \\
\hline 46.70 & 0.221918 & 2800 & 0.598948 & 0.654257 & 0.709567 \\
\hline 59.32 & 0.221918 & 2850 & 0.278939 & 0.334248 & 0.389558 \\
\hline 63.55 & 0.221918 & 2860 & 0.215611 & 0.270920 & 0.326230 \\
\hline 64.70 & 0.221918 & 2865 & 0.184030 & 0.239340 & 0.294649 \\
\hline 46.60 & 0.298630 & 2750 & 0.847874 & 0.912035 & 0.976196 \\
\hline 57.50 & 0.298630 & 2800 & 0.567041 & 0.631203 & 0.695364 \\
\hline
\end{tabular}

TABLe 7: Pricing results by our pricing formula and the Black-Scholes model for call option.

\begin{tabular}{lcccccc}
\hline$K$ & $\tau$ & $d_{1}$ & $d_{2}$ & $d_{3}$ & $C$ & $C_{1}$ \\
\hline 2870 & 0.043836 & 0.036511 & 0.061093 & 0.085675 & 21.05 & 28.26 \\
2875 & 0.043836 & -0.034298 & -0.009716 & 0.014866 & 18.15 & 25.85 \\
2880 & 0.043836 & -0.104985 & -0.080403 & -0.055821 & 18.20 & 23.58 \\
2890 & 0.043836 & -0.245990 & -0.221408 & -0.196826 & 13.70 & 19.45 \\
2900 & 0.043836 & -0.386509 & -0.361927 & -0.337345 & 8.50 & 15.87 \\
2870 & 0.123288 & 0.103532 & 0.144758 & 0.185983 & 45.00 & 50.06 \\
2875 & 0.123288 & 0.061310 & 0.102535 & 0.143760 & 40.85 & 47.25 \\
\hline
\end{tabular}


TABle 7: Continued.

\begin{tabular}{|c|c|c|c|c|c|c|c|}
\hline$K$ & $\tau$ & $d_{1}$ & $d_{2}$ & $d_{3}$ & $C$ & $C_{1}$ & $C_{2}$ \\
\hline 2895 & 0.123288 & -0.106850 & -0.065625 & -0.024399 & 31.68 & 38.36 & 36.73 \\
\hline 2900 & 0.123288 & -0.148708 & -0.107483 & -0.066258 & 29.50 & 36.27 & 33.49 \\
\hline 2925 & 0.123288 & -0.356924 & -0.315698 & -0.274473 & 18.10 & 27.00 & 19.26 \\
\hline 2940 & 0.123288 & -0.481000 & -0.439775 & -0.398549 & 14.10 & 22.35 & 12.25 \\
\hline 2950 & 0.123288 & -0.563367 & -0.522141 & -0.480916 & 11.50 & 19.61 & 8.19 \\
\hline 2955 & 0.123288 & -0.604445 & -0.563220 & -0.521995 & 10.45 & 18.34 & 6.35 \\
\hline 2870 & 0.219178 & 0.151657 & 0.206624 & 0.261591 & 64.00 & 69.14 & 75.39 \\
\hline 2875 & 0.219178 & 0.119990 & 0.174957 & 0.229924 & 63.80 & 66.58 & 71.78 \\
\hline 2900 & 0.219178 & -0.037523 & 0.017444 & 0.072411 & 47.50 & 54.73 & 54.98 \\
\hline 2950 & 0.219178 & -0.348517 & -0.293550 & -0.238583 & 26.75 & 35.58 & 27.81 \\
\hline 3000 & 0.219178 & -0.654284 & -0.599317 & -0.544350 & 13.30 & 21.94 & 8.99 \\
\hline 3025 & 0.219178 & -0.805261 & -0.750294 & -0.695327 & 8.75 & 16.88 & 2.42 \\
\hline 2900 & 0.295890 & 0.018662 & 0.082528 & 0.146394 & 62.77 & 67.32 & 69.15 \\
\hline 2900 & 0.372603 & 0.062039 & 0.133708 & 0.205376 & 76.38 & 78.74 & 81.78 \\
\hline 3100 & 0.372603 & -0.868516 & -0.796848 & -0.725179 & 9.40 & 18.99 & 3.50 \\
\hline 2900 & 0.468493 & 0.105947 & 0.186310 & 0.266673 & 91.40 & 91.87 & 96.10 \\
\hline 3000 & 0.468493 & -0.315908 & -0.235545 & -0.155182 & 41.26 & 52.22 & 44.38 \\
\hline 3100 & 0.468493 & -0.723929 & -0.643566 & -0.563203 & 15.00 & 27.13 & 12.45 \\
\hline
\end{tabular}

TABLE 8: Pricing results by our pricing formula and the Black-Scholes model for put option.

\begin{tabular}{|c|c|c|c|c|c|c|c|}
\hline$K$ & $\tau$ & $d_{1}$ & $d_{2}$ & $d_{3}$ & $P$ & $P_{1}$ & $P_{2}$ \\
\hline 2550 & 0.043836 & 4.845658 & 4.870240 & 4.894822 & 0.59 & 0.00 & 0.05 \\
\hline 2575 & 0.043836 & 4.448776 & 4.473358 & 4.497940 & 0.70 & 0.00 & 0.14 \\
\hline 2600 & 0.043836 & 4.055729 & 4.080311 & 4.104893 & 0.81 & 0.00 & 0.35 \\
\hline 2650 & 0.043836 & 3.280846 & 3.305428 & 3.330010 & 1.20 & 0.01 & 1.59 \\
\hline 2660 & 0.043836 & 3.127625 & 3.152207 & 3.176790 & 1.40 & 0.02 & 2.08 \\
\hline 2675 & 0.043836 & 2.898871 & 2.923453 & 2.948035 & 1.64 & 0.04 & 3.02 \\
\hline 2700 & 0.043836 & 2.520449 & 2.545031 & 2.569613 & 1.95 & 0.14 & 5.27 \\
\hline 2300 & 0.123288 & 5.474086 & 5.515311 & 5.556536 & 1.30 & 0.00 & 0.01 \\
\hline 2400 & 0.123288 & 4.441720 & 4.482946 & 4.524171 & 1.95 & 0.00 & 0.14 \\
\hline 2500 & 0.123288 & 3.451504 & 3.492730 & 3.533955 & 3.26 & 0.01 & 1.11 \\
\hline 2590 & 0.123288 & 2.593606 & 2.634832 & 2.676057 & 5.40 & 0.20 & 4.65 \\
\hline 2600 & 0.123288 & 2.500131 & 2.541356 & 2.582581 & 6.00 & 0.27 & 5.32 \\
\hline 2615 & 0.123288 & 2.360589 & 2.401814 & 2.443040 & 6.20 & 0.41 & 6.45 \\
\hline 2625 & 0.123288 & 2.268005 & 2.309230 & 2.350456 & 7.10 & 0.53 & 7.29 \\
\hline 2670 & 0.123288 & 1.855696 & 1.896922 & 1.938147 & 9.20 & 1.62 & 11.98 \\
\hline 2675 & 0.123288 & 1.810314 & 1.851539 & 1.892764 & 9.40 & 1.82 & 12.60 \\
\hline 2700 & 0.123288 & 1.584666 & 1.625892 & 1.667117 & 11.50 & 3.13 & 15.93 \\
\hline 2725 & 0.123288 & 1.361099 & 1.402324 & 1.443549 & 14.30 & 5.16 & 19.69 \\
\hline 2775 & 0.123288 & 0.920051 & 0.961277 & 1.002502 & 20.50 & 12.41 & 28.36 \\
\hline 2800 & 0.123288 & 0.702499 & 0.743724 & 0.784950 & 25.85 & 18.18 & 33.26 \\
\hline 2825 & 0.123288 & 0.486881 & 0.528106 & 0.569331 & 31.50 & 25.71 & 38.61 \\
\hline 2850 & 0.123288 & 0.273162 & 0.314387 & 0.355612 & 39.00 & 35.19 & 44.52 \\
\hline 2200 & 0.219178 & 4.988269 & 5.043237 & 5.098204 & 2.60 & 0.00 & 0.03 \\
\hline 2400 & 0.219178 & 3.405298 & 3.460265 & 3.515232 & 5.80 & 0.02 & 1.16 \\
\hline 2500 & 0.219178 & 2.662636 & 2.717603 & 2.772570 & 9.00 & 0.22 & 4.11 \\
\hline 2540 & 0.219178 & 2.373857 & 2.428824 & 2.483791 & 11.00 & 0.54 & 6.28 \\
\hline 2550 & 0.219178 & 2.302373 & 2.357340 & 2.412307 & 11.95 & 0.67 & 6.94 \\
\hline 2575 & 0.219178 & 2.124882 & 2.179849 & 2.234816 & 13.20 & 1.10 & 8.80 \\
\hline 2600 & 0.219178 & 1.949106 & 2.004073 & 2.059040 & 14.80 & 1.76 & 10.99 \\
\hline 2650 & 0.219178 & 1.602568 & 1.657535 & 1.712502 & 19.95 & 4.13 & 16.46 \\
\hline 2725 & 0.219178 & 1.094832 & 1.149799 & 1.204766 & 29.92 & 12.23 & 27.74 \\
\hline 2825 & 0.219178 & 0.439168 & 0.494135 & 0.549103 & 51.10 & 37.78 & 49.91 \\
\hline 2850 & 0.219178 & 0.278879 & 0.333846 & 0.388814 & 58.00 & 47.68 & 57.10 \\
\hline 2865 & 0.219178 & 0.183379 & 0.238346 & 0.293314 & 65.20 & 54.36 & 61.80 \\
\hline 2425 & 0.295890 & 2.819513 & 2.883379 & 2.947245 & 10.76 & 0.16 & 3.16 \\
\hline 2500 & 0.295890 & 2.342589 & 2.406455 & 2.470321 & 14.75 & 0.70 & 6.54 \\
\hline 2675 & 0.295890 & 1.283205 & 1.347071 & 1.410937 & 32.50 & 9.91 & 24.37 \\
\hline 2850 & 0.295890 & 0.290978 & 0.354844 & 0.418710 & 69.79 & 55.32 & 64.91 \\
\hline 2860 & 0.295890 & 0.236134 & 0.300000 & 0.363866 & 76.22 & 59.70 & 68.22 \\
\hline
\end{tabular}


The $t$-statistics equals -4.4919 with degree of freedom 38 , and its $p$ value is 0.00019 . Thus, we accept $H_{1}$, i.e., $E\left(\left|P_{2}-P\right|\right)<E\left(\left|P_{1}-P\right|\right)$. That is, the prices of put options computed by Theorem 3 are far nearer to their actual prices than those computed by the Black-Scholes model.

Therefore, our pricing formulas in Theorems 2 and 3 have less absolute errors than those of the Black-Scholes model for both call options and put options. That is, the fitting effect of our pricing formulas is superior to that of the Black-Scholes model.

\section{Conclusion}

In this study, we obtain the pricing formula of European options, including European call option and European put option, in a risk-aversive market. Corollaries of our obtained results improve the Black-Scholes model owning to its much weaker conditions. It follows from our obtained results that European option value depends on the drift coefficient $\mu$ of its underlying security, which does not display in the Black-Scholes model only because $\mu=r$ in a risk-neutral financial market according to the no-arbitrage opportunity principle. Empirical analyses show that the fitting effect of our pricing formulas is superior to that of the Black-Scholes model.

\section{Appendix}

\section{A. Proof of Theorem 2}

In order to prove Theorem 2, we need the following lemma.

Lemma A.1. Assuming $\ln X \sim N\left(\mu, \sigma^{2}\right)$, then for any real number $m \in \mathbb{R}$ and positive real number $K \in \mathbb{R}^{+}$, it follows that

$$
E\left[X^{m} 1_{\{X \geq K\}}\right]=e^{m \mu+(1 / 2) m^{2} \sigma^{2}} \Phi\left(\frac{1}{\sigma}\left(\ln \frac{1}{K}+\mu+m \sigma^{2}\right)\right) .
$$

Proof. If $\ln X \sim N\left(\mu, \sigma^{2}\right)$, denote the density function of $X$ by $f(x ; \mu, \sigma)$, and then

$$
f(x ; \mu, \sigma)= \begin{cases}\frac{1}{x \sigma \sqrt{2 \pi}} \exp \left\{-\frac{(\ln x-\mu)^{2}}{2 \sigma^{2}}\right\}, & x>0, \\ 0, & x \leq 0 .\end{cases}
$$

For any real number $m \in \mathbb{R}$ and positive real number $K \in \mathbb{R}^{+}$, it follows that

$$
\begin{aligned}
E\left[X^{m} 1_{\{X \geq K\}}\right]= & \int_{-\infty}^{+\infty} x^{m} 1_{\{x \geq K\}} f\left(x ; \mu, \sigma^{2}\right) \mathrm{d} x, \\
& =\int_{K}^{+\infty} x^{m} \frac{1}{x \sigma \sqrt{2 \pi}} \exp \left\{-\frac{(\ln x-\mu)^{2}}{2 \sigma^{2}}\right\} \mathrm{d} x \\
& =\int_{\ln K}^{+\infty} e^{m y} \frac{1}{\sigma \sqrt{2 \pi}} \exp \left\{-\frac{(y-\mu)^{2}}{2 \sigma^{2}}\right\} \mathrm{d} y \\
& =e^{m \mu+(1 / 2) m^{2} \sigma^{2}} \int_{\ln K}^{+\infty} \frac{1}{\sigma \sqrt{2 \pi}} \exp \\
& \left\{-\frac{\left[y-\left(\mu+m \sigma^{2}\right)\right]^{2}}{2 \sigma^{2}}\right\} \mathrm{d} y \\
& =e^{m \mu+(1 / 2) m^{2} \sigma^{2}} \int_{1 / \sigma\left[\ln K-\left(\mu+m \sigma^{2}\right)\right]}^{+\infty} \frac{1}{\sqrt{2 \pi}} \exp \left\{-\frac{z^{2}}{2}\right\} \mathrm{d} z \\
& =e^{m \mu+(1 / 2) m^{2} \sigma^{2}}\left(1-\Phi\left(\frac{1}{\sigma}\left[\ln K-\left(\mu+m \sigma^{2}\right)\right]\right)\right) \\
& =e^{m \mu+(1 / 2) m^{2} \sigma^{2}} \Phi\left(\frac{1}{\sigma}\left(\ln \frac{1}{K}+\mu+m \sigma^{2}\right)\right) .
\end{aligned}
$$

The proof is complete.

Noting that the underlying asset follows a geometric Brownian motion with drift coefficient $\mu$ and diffusion coefficient $\sigma>0$ and the current price of underlying asset is $S_{0}$, it follows from It $\widehat{o}$ formula that

$$
S_{\tau}=S_{0} \exp \left\{\left(\mu-\frac{1}{2} \sigma^{2}\right) \tau+\sigma W_{\tau}\right\},
$$

where $W=\left\{W_{t}, t \geq 0\right\}$ is the standard Wiener process, so

$$
\ln S_{\tau} \sim N\left(\ln S_{0}+\left(\mu-\frac{1}{2} \sigma^{2}\right) \tau, \sigma^{2} \tau\right) \text {. }
$$

It yields from Lemma A.1 and (A.5) that

$$
\begin{aligned}
E\left[1_{\left\{S_{\tau} \geq K\right\}}\right] & =\Phi\left(d_{1}\right), \\
E\left[S_{\tau} 1_{\left\{S_{\tau} \geq K\right\}}\right] & =S_{0} e^{\mu \tau} \Phi\left(d_{2}\right), \\
E\left[S_{\tau}^{2} 1_{\left\{S_{\tau} \geq K\right\}}\right] & =S_{0}^{2} e^{\left(\mu+\sigma^{2}\right) \tau} \Phi\left(d_{3}\right) .
\end{aligned}
$$

It follows from (A.6) to (A.8) that 


$$
\begin{aligned}
E\left[\left(S_{\tau}-K\right)^{+}\right]= & E\left[\left(S_{\tau}-K\right) 1_{\left\{S_{\tau} \geq K\right\}}\right], \\
= & E\left[S_{\tau} 1_{\left\{S_{\tau} \geq K\right\}}\right]-K E\left[1_{\left\{S_{\tau} \geq K\right\}}\right] \\
= & S_{0} e^{\mu \tau} \Phi\left(d_{2}\right)-K \Phi\left(d_{1}\right), \\
E\left[\left(\left(S_{\tau}-K\right)^{+}\right)^{2}\right]= & E\left[\left(S_{\tau}-K\right)^{2} 1_{\left\{S_{\tau} \geq K\right\}}\right], \\
= & E\left[S_{\tau}^{2} 1_{\left\{S_{\tau} \geq K\right\}}\right]-2 K E\left[S_{\tau} 1_{\left\{S_{\tau} \geq K\right\}}\right] \\
& +K^{2} E\left[1_{\left\{S_{\tau} \geq K\right\}}\right] \\
= & S_{0}^{2} e^{\left(\mu+\sigma^{2}\right) \tau} \Phi\left(d_{3}\right)-2 K S_{0} e^{\mu \tau} \Phi\left(d_{2}\right) \\
& +K^{2} \Phi\left(d_{1}\right) .
\end{aligned}
$$

Furthermore, it obtains from (A.9) and (A.10) that

$$
\begin{aligned}
\operatorname{std}\left(\left(S_{\tau}-K\right)^{+}\right) & =\operatorname{sqrt}\left\{E\left[\left(\left(S_{\tau}-K\right)^{+}\right)^{2}\right]-E^{2}\left[\left(S_{\tau}-K\right)^{+}\right]\right\} \\
& =\operatorname{sqrt}\left\{S_{0}^{2} e^{2 \mu \tau}\left(e^{\sigma^{2} \tau} \Phi\left(d_{3}\right)-\Phi^{2}\left(d_{2}\right)\right)-K S_{0} e^{\mu \tau} \Phi\left(d_{2}\right) \Phi\left(-d_{1}\right)+K^{2} \Phi\left(d_{1}\right) \Phi\left(-d_{1}\right)\right\}
\end{aligned}
$$

Thus, it yields from Proposition 1, (A.9), and (A.11) that

$$
\begin{aligned}
C\left(S_{0}, K, r, \sigma, \tau, \lambda\right)= & e^{-r \tau}\left\{E\left[\left(S_{\tau}-K\right)^{+}\right]+\lambda \cdot \operatorname{std}\left(\left(S_{\tau}-K\right)^{+}\right)\right\} \\
= & S_{0} e^{(\mu-r) \tau} \Phi\left(d_{2}\right)-K e^{-r \tau} \Phi\left(d_{1}\right)+\lambda e^{-r \tau} \cdot \operatorname{sqrt}\left\{S_{0}^{2} e^{2 \mu \tau}\left(e^{\sigma^{2} \tau} \Phi\left(d_{3}\right)-\Phi^{2}\left(d_{2}\right)\right)-K S_{0} e^{\mu \tau} \Phi\left(d_{2}\right) \Phi\left(-d_{1}\right)\right. \\
& \left.+K^{2} \Phi\left(d_{1}\right) \Phi\left(-d_{1}\right)\right\} .
\end{aligned}
$$

The proof is complete.

$$
E\left[X^{m} 1_{\{X \leq K\}}\right]=e^{m \mu+(1 / 2) m^{2} \sigma^{2}} \Phi\left(-\frac{1}{\sigma}\left(\ln \frac{1}{K}+\mu+m \sigma^{2}\right)\right) .
$$

\section{B. Proof of Theorem 3}

In order to prove Theorem 3, we first present another lemma as follows.

Lemma B.1. Assuming $\ln X \sim N\left(\mu, \sigma^{2}\right)$, then for any real number $m \in \mathbb{R}$ and positive real number $K \in \mathbb{R}^{+}$, it follows that
Proof. If $\ln X \sim N\left(\mu, \sigma^{2}\right)$, denote the density function of $X$ by $f(x ; \mu, \sigma)$, and then

$$
f(x ; \mu, \sigma)= \begin{cases}\frac{1}{x \sigma \sqrt{2 \pi}} \exp \left\{-\frac{(\ln x-\mu)^{2}}{2 \sigma^{2}}\right\}, & x>0, \\ 0, & x \leq 0 .\end{cases}
$$


For any real number $m \in \mathbb{R}$ and positive real number $K \in \mathbb{R}^{+}$, it follows that

$$
\begin{aligned}
E\left[X^{m} 1_{\{X \leq K\}}\right] & =\int_{-\infty}^{+\infty} x^{m} 1_{\{x \leq K\}} f\left(x ; \mu, \sigma^{2}\right) \mathrm{d} x \\
& =\int_{-\infty}^{K} x^{m} \frac{1}{x \sigma \sqrt{2 \pi}} \exp \left\{-\frac{(\ln x-\mu)^{2}}{2 \sigma^{2}}\right\} \mathrm{d} x \\
& =\int_{-\infty}^{\ln K} e^{m y} \frac{1}{\sigma \sqrt{2 \pi}} \exp \left\{-\frac{(y-\mu)^{2}}{2 \sigma^{2}}\right\} \mathrm{d} y \\
& =e^{m \mu+(1 / 2) m^{2} \sigma^{2}} \int_{-\infty}^{\ln K} e^{m y} \frac{1}{\sigma \sqrt{2 \pi}} \exp \\
& \left\{-\frac{\left[y-\left(\mu+m \sigma^{2}\right)\right]^{2}}{2 \sigma^{2}}\right\} \mathrm{d} y \\
& =e^{m \mu+(1 / 2) m^{2} \sigma^{2}} \int_{-\infty}^{1 / \sigma\left[\ln K-\left(\mu+m \sigma^{2}\right)\right]} \frac{1}{\sqrt{2 \pi}} \exp \left\{-\frac{z^{2}}{2}\right\} \mathrm{d} z \\
& =e^{m \mu+(1 / 2) m^{2} \sigma^{2}} \Phi\left(\frac{1}{\sigma}\left[\ln K-\left(\mu+m \sigma^{2}\right)\right]\right) \\
& =e^{m \mu+(1 / 2) m^{2} \sigma^{2}} \Phi\left(-\frac{1}{\sigma}\left(\ln \frac{1}{K}+\mu+m \sigma^{2}\right)\right) .
\end{aligned}
$$

The proof is complete.

Noting that the underlying asset follows a geometric Brownian motion with drift coefficient $\mu$ and diffusion coefficient $\sigma>0$ and the current price of underlying asset is $S_{0}$, it follows from Itô formula that

$$
S_{\tau}=S_{0} \exp \left\{\left(\mu-\frac{1}{2} \sigma^{2}\right) \tau+\sigma W_{\tau}\right\}
$$

where $W=\left\{W_{t}, t \geq 0\right\}$ is a standard Wiener process, so

$$
\ln S_{\tau} \sim N\left(\ln S_{0}+\left(\mu-\frac{1}{2} \sigma^{2}\right) \tau, \sigma^{2} \tau\right) \text {. }
$$

It yields from Lemma B.1 and (B.5) that

$$
\begin{aligned}
E\left[1_{\left\{S_{\tau} \leq K\right\}}\right] & =\Phi\left(-d_{1}\right), \\
E\left[S_{\tau} 1_{\left\{S_{\tau} \leq K\right\}}\right] & =S_{0} e^{\mu \tau} \Phi\left(-d_{2}\right), \\
E\left[S_{\tau}^{2} 1_{\left\{S_{\tau} \leq K\right\}}\right] & =S_{0}^{2} e^{\left(\mu+\sigma^{2}\right) \tau} \Phi\left(-d_{3}\right) .
\end{aligned}
$$

It follows from (B.6) to (B.8) that

$$
\begin{aligned}
E\left[\left(K-S_{\tau}\right)^{+}\right]= & E\left[\left(K-S_{\tau}\right) 1_{\left\{S_{\tau} \leq K\right\}}\right] \\
= & K E\left[1_{\left\{S_{\tau} \leq K\right\}}\right]-E\left[S_{\tau} 1_{\left\{S_{\tau} \leq K\right\}}\right] \\
= & K \Phi\left(-d_{1}\right)-S_{0} e^{\mu \tau} \Phi\left(-d_{2}\right) \\
E\left[\left(\left(K-S_{\tau}\right)^{+}\right)^{2}\right]= & E\left[\left(K-S_{\tau}\right)^{2} 1_{\left\{S_{\tau} \leq K\right\}}\right] \\
= & E\left[S_{\tau}^{2} 1_{\left\{S_{\tau} \leq K\right\}}\right]-2 K E\left[S_{\tau} 1_{\left\{S_{\tau} \leq K\right\}}\right] \\
& +K^{2} E\left[1_{\left\{S_{\tau} \leq K\right\}}\right] \\
= & S_{0}^{2} e^{\left(\mu+\sigma^{2}\right) \tau} \Phi\left(-d_{3}\right)-2 K S_{0} e^{\mu \tau} \Phi\left(-d_{2}\right) \\
& +K^{2} \Phi\left(-d_{1}\right) .
\end{aligned}
$$

Furthermore, it obtains from (B.9) and (B.10) that

$$
\begin{aligned}
\operatorname{std}\left(\left(K-S_{\tau}\right)^{+}\right) & =\operatorname{sqrt}\left\{E\left[\left(\left(K-S_{\tau}\right)^{+}\right)^{2}\right]-E^{2}\left[\left(K-S_{\tau}\right)^{+}\right]\right\} \\
& =\operatorname{sqrt}\left\{S_{0}^{2} e^{2 \mu \tau}\left(e^{\sigma^{2} \tau} \Phi\left(-d_{3}\right)-\Phi^{2}\left(-d_{2}\right)\right)-K S_{0} e^{\mu \tau} \Phi\left(-d_{2}\right) \Phi\left(d_{1}\right)+K^{2} \Phi\left(-d_{1}\right) \Phi\left(d_{1}\right)\right\}
\end{aligned}
$$

Thus, it yields from (B.9) and (B.11) that

$$
\begin{aligned}
P\left(S_{0}, K, r, \sigma, \tau, \lambda\right)= & e^{-r \tau}\left\{E\left[\left(K-S_{\tau}\right)^{+}\right]+\lambda \cdot \operatorname{std}\left(\left(K-S_{\tau}\right)^{+}\right)\right\} \\
= & K e^{-r \tau} \Phi\left(-d_{1}\right)-S_{0} e^{(\mu-r) \tau} \Phi\left(-d_{2}\right)+\lambda e^{-r \tau} \cdot \operatorname{sqrt}\left\{S_{0}^{2} e^{2 \mu \tau}\left(e^{\sigma^{2} \tau} \Phi\left(-d_{3}\right)-\Phi^{2}\left(-d_{2}\right)\right)-K S_{0} e^{\mu \tau} \Phi\left(-d_{2}\right) \Phi\left(d_{1}\right)\right. \\
& \left.+K^{2} \Phi\left(-d_{1}\right) \Phi\left(d_{1}\right)\right\} .
\end{aligned}
$$

The proof is complete. 


\section{Data Availability}

The data used to support the findings of this study are available from the corresponding author upon request.

\section{Conflicts of Interest}

The authors declare that they have no conflicts of interest.

\section{Acknowledgments}

This study was supported by the National Natural Science Foundation of China (no. 11471120).

\section{References}

[1] F. Black and M. Scholes, "The pricing of options and corporate liabilities," Journal of Political Economy, vol. 81, no. 3, pp. 637-654, 1973.

[2] R. C. Merton, "Theory of rational option pricing," The Bell Journal of Economics and Management Science, vol. 4, no. 1, pp. 141-183, 1973.

[3] G. Bakshi, C. Cao, and Z. Chen, "Empirical performance of alternative option pricing models," The Journal of Finance, vol. 52, no. 5, pp. 2003-2049, 1997.

[4] N. Gârleanu, L. H. Pedersen, and A. M. Poteshman, "Demand-based option pricing," The Review of Financial Studies, vol. 22, no. 10, pp. 4259-4299, 2009.

[5] N. Cai and S. G. Kou, "Option pricing under a mixed-exponential jump diffusion model," Management Science, vol. 57, no. 11, pp. 2067-2081, 2011.

[6] C. Bernarda and C. Czadob, "Multivariate option pricing using copulae," Applied Stochastic Models in Business and Industry, vol. 29, pp. 509-526, 2013.

[7] C. Bandi and D. Bertsimas, "Robust option pricing," European Journal of Operational Research, vol. 239, no. 3, pp. 842-853, 2014.

[8] S. Bao, S. Chen, W. A. Zheng, and Y. Zhou, "Forecasting semistationary processes and statistical arbitrage," Statistical Theory and Related Fields, vol. 4, no. 2, pp. 179-189, 2020.

[9] E. Moretto, S. Pasquali, and B. Trivellato, "Option pricing under deformed Gaussian distributions," Physica A: Statistical Mechanics and its Applications, vol. 446, pp. 246-263, 2016.

[10] M. Leippold and S. Schärer, "Discrete-time option pricing with stochastic liquidity," Journal of Banking \& Finance, vol. 75, pp. 1-16, 2017.

[11] J. Hok and T. L. Chan, "Option pricing with Legendre polynomials," Journal of Computational and Applied Mathematics, vol. 322, pp. 25-45, 2017.

[12] A. H. Davison and S. Mamba, "Symmetry methods for option pricing," Communications in Nonlinear Science and Numerical Simulation, vol. 47, pp. 421-425, 2017.

[13] S. Willems, "Asian option pricing with orthogonal polynomials," Quantitative Finance, vol. 19, no. 4, pp. 605-618, 2019.

[14] Y. Liu, X. Chen, and D. A. Ralescu, "Uncertain currency model and currency option pricing," International Journal of Intelligent Systems, vol. 30, no. 1, pp. 40-51, 2015.

[15] P. Friz, S. Gerhold, and A. Pinter, "Option pricing in the moderate deviations regime," Mathematical Finance, vol. 28, no. 3, pp. 962-988, 2018.

[16] A. Dubinsky, M. Johannes, A. Kaeck, and N. J. Seeger, "Option pricing of earnings announcement risks," The Review of Financial Studies, vol. 32, no. 2, pp. 646-687, 2019.
[17] J. Huh, "Pricing options with exponential Lévy neural network," Expert Systems With Applications, vol. 127, pp. 128140, 2019.

[18] X. Liu, Y. Cao, C. Ma, and L. Shen, "Wavelet-based option pricing: an empirical study," European Journal of Operational Research, vol. 272, no. 3, pp. 1132-1142, 2019.

[19] H. Siddiqi, "Anchoring-adjusted option pricing models," Journal of Behavioral Finance, vol. 20, no. 2, pp. 139-153, 2019. 